\title{
LDL delivery of microbial small RNAs drives atherosclerosis through macrophage TLR8
}

Ryan Allen ( $\nabla$ ryan.m.allen@vumc.org)

Vanderbilt University Medical Center https://orcid.org/0000-0002-8057-3436

Danielle Michell

Vanderbilt University Medical Center

Ashley Cavnar

Vanderbilt University Medical Center

Wanying Zhu

Vanderbilt University Medical Center

Neil Makhijani

Vanderbilt University Medical Center

Danielle Contreras

Vanderbilt University Medical Center

Chase Raby

Vanderbilt University Medical Center

Elizabeth Semler

Vanderbilt University Medical Center

Carlisle DeJulius

Vanderbilt University

Mark Castleberry

Vanderbilt University Medical Center

Youmin Zhang

Vanderbilt University Medical Center

Marisol Ramirez

Vanderbilt University Medical Center

Shilin Zhao

Vanderbilt University Medical Center

Craig Duvall

Vanderbilt University

Amanda Doran

Vanderbilt University Medical Center

Quanhu Sheng

Vanderbilt University Medical Center

MacRae Linton 
Vanderbilt University Medical Center

Kasey Vickers

Vanderbilt University Medical Center

\section{Article}

Keywords: LDL, small RNA, macrophage TLR8

Posted Date: January 7th, 2021

DOI: https://doi.org/10.21203/rs.3.rs-134271/v1

License: (c) (i) This work is licensed under a Creative Commons Attribution 4.0 International License. Read Full License

Version of Record: A version of this preprint was published at Nature Cell Biology on December 6th, 2022. See the published version at https://doi.org/10.1038/s41556-022-01030-7. 


\section{Abstract}

Macrophages present a spectrum of phenotypes that mediate both the pathogenesis and resolution of atherosclerotic lesions. Inflammatory macrophage phenotypes are pro-atherogenic, but the natural factors that instigate this polarization are largely unknown. Here, we demonstrate that microbial small RNAs (msRNA) are enriched on LDL and drive pro-inflammatory macrophage polarization and cytokine secretion via activation of the ribonucleic acid sensor toll-like receptor 8 (TLR8). Removal of msRNA cargo during LDL re-constitution yields particles that readily promote sterol loading but fail to stimulate inflammatory activation. Competitive antagonism of TLR8 with non-targeting locked nucleic acids (ntLNA) was found to prevent $\mathrm{nLDL}$-induced macrophage polarization in vitro, and re-organize lesion macrophage phenotypes in vivo, as determined by single-cell RNA sequencing. Critically, this was associated with reduced disease burden in distinct mouse models of atherosclerosis. These results identify LDL-msRNA as instigators of atherosclerosis-associated inflammation and support alternative functions of LDL beyond cholesterol transport.

\section{Introduction}

Atherosclerosis is a pathophysiological process driven by dyslipidemia and unchecked, chronic inflammation ${ }^{1,2}$. Pharmacological lowering of LDL-C levels is a primary clinical target to reduce the risk of cardiovascular disease (CVD); yet, residual risk remains for many with properly managed plasma lipid levels ${ }^{3}$. Results of the CANTOS trial support that targeting inflammation - monoclonal antibody (Canakinumab) against interleukin $1 \beta(I L-1 \beta)$ - in the absence of reducing plasma lipids is a viable approach to mitigating cardiovascular events ${ }^{4}$. Nonetheless, inhibiting facilitators of systemic immune responses (i.e. cytokines), may be problematic for susceptible patients. A more desirable approach may be to target upstream, inciting stimuli that promote atherosclerosis-associated inflammation; however, identification of instigating factors has proven elusive.

Macrophages have long been the focus of atherosclerosis research due to their ability to enhance inflammation, recruit immune cells, and propensity to become engorged with cholesterol to yield foam cells ${ }^{5}$. Macrophage scavenger receptor-mediated uptake of modified (e.g. oxidized) LDL is widely regarded as a crucial event in foam cell formation within atheroma ${ }^{6}$. The importance of both these receptors and the impact of LDL modifications have been accentuated by genetic studies in mice ${ }^{7-10}$, and coordinated signaling between scavenger receptors and pro-inflammatory cell signaling macrophages has been reported in atherosclerosis ${ }^{11,12}$. Nonetheless, the inability of anti-oxidation therapies to improve clinical outcomes in CVD has introduced questions as to whether LDL oxidation is a cause or consequence of atherosclerosis ${ }^{13,14}$. Furthermore, single-cell RNA-sequencing (scRNA-seq) studies have found that transcriptomes of lipid-loaded foam cells within the atheroma are not associated with inflammatory phenotypes ${ }^{15-17}$, supporting earlier studies that activation of sterol-sensing machinery suppresses inflammatory gene expression ${ }^{18}$. Intriguingly, small native LDL (nLDL) particles, which carry reduced sterol cargo, are associated with increased risk of CVD ${ }^{19}$. Collectively, these studies indicate that 
a cholesterol-independent mechanism may link nLDL to the chronic inflammation that underlies atherosclerosis and CVD.

\section{Results}

nLDL activates pro-inflammatory gene expression and cytokine secretion in macrophages. A causal role for $\mathrm{nLDL}$ in atherosclerosis has been largely overlooked; however, $\mathrm{nLDL}$ particles can accumulate in human atherosclerotic lesions to concentrations $>2 \mathrm{mg} / \mathrm{ml}^{20}$. At these patho-physiological levels, $\mathrm{nLDL}$ uptake by macrophages likely occurs through fluid-phase pinocytosis in a time- and concentrationdependent manner ${ }^{21,22}$. To determine the impact of $n L D L$ on macrophage activation, phorbol-12myristate-13-acetate (PMA)-differentiated human THP-1 macrophages were treated with physiological concentrations of human $\mathrm{nLDL}(0.5 \mathrm{mg} / \mathrm{ml})$ and gene (mRNA) expression changes were quantified by high-throughput RNA sequencing (RNA-seq). In total, 365 up-regulated genes and 141 down-regulated genes were observed to be significantly altered by $n L D L$ treatments (Fig. 1a; Supplementary Table 1). Network analysis of nLDL-altered transcripts identified enrichment for multiple pro-inflammatory transcription factors, including nuclear factor kappa B (NF-KB) and C/EBPb (Fig. 1b,c; Supplementary Tables 2,3). nLDL-induced changes to NF-KB and C/EBPb transcriptional target gene expression were quantified in dose and temporal studies by qPCR for mRNA levels (Fig. 1d and Supplementary Fig. 1) and multiplex ELISA for secreted cytokines (Fig. 1e). nLDL treatments were found to result in the accumulation of APOB protein in macrophages and the formation of lipid-droplets, as evidenced by the stabilization of PLIN2 protein (Fig. 1 f and Supplementary Fig. 1), observations that are supported by previous studies ${ }^{22,23}$. Intracellular increases of IL-1b protein and inflammatory cell signaling factors, IKKb and IKBa, - transcriptional targets of NF-kB - further validated RNA-seq results (Fig. 1f). Consistent with observations in human cells, similar results were obtained by exposing primary bone-marrow derived macrophages (BMDM) to nLDL (Supplementary Fig. 1). Collectively, these data support that $\mathrm{LDL}$ activates macrophage cytokine expression and secretion likely through NF-KB.

\section{nLDL activates the single-stranded RNA-sensing TLR8 in macrophages.}

Macrophage toll-like receptors (TLR) facilitate innate immunity by recognizing pathogen-associated molecular patterns (PAMPs), which have been extensively studied in atherosclerosis ${ }^{24}$. Extracellular PAMPs are detected by surface TLRs (TLR1, $-2,-4,-5,-6)$, whereas intracellular PAMPs (i.e. nucleic acids) are recognized by endosomal TLRs (TLR3, $-7,-8,-9)$. To determine whether nLDL-induced macrophage activation was through surface or endosomal TLRs, THP-1 macrophages were pre-treated with bafilomycin $A 1$, a vacuolar proton pump inhibitor that disrupts endo-lysosome maturation, prior to treatment with $\mathrm{nLDL}$, lipopolysaccharide (LPS; TLR4 ligand), or single-stranded RNA (ssRNA) oligonucleotide (sSRNA40; TLR8 ligand). Global TLR signaling was also disrupted using a cell-permeable calcium chelator (EGTA-AM) prior to treatments ${ }^{25}$. Bafilomycin treatment was found to reduce $\mathrm{nLDL}$ - and ssRNA40-, but not LPS-mediated secretion of TNF-a (Fig. 2a), supporting the involvement of an endosomal TLR in nLDL-induced activation of NF-kB. To further resolve this, individual TLRs were co- 
expressed in HEK293T cells with an NF-KB promoter-driven luciferase reporter and validated by treatment with TLR-specific ligands, or TNF-a for cells expressing no TLR (Fig. 2b). Strikingly, we observed activation of nLDL-driven NF-KB activity only in the presence of TLR8, an endosomal receptor that recognizes SSRNA and products of ssRNA catabolism ${ }^{26,27}$. These observations were confirmed to be specific for TLR8, but not TLR7, indicating that TLR8 alone is sufficient for nLDL-induced NF-KB activity (Fig. 2c). Partial silencing of TLR8 with siRNAs blunted nLDL-induced cytokine expression and secretion of TNF-a and IL-6 in macrophages (Fig. 2e and 2e). Furthermore, when microparticles containing the selective TLR8 inhibitor CU-CPT9a ${ }^{28}$ were given to THP-1 macrophages we observed a significant reduction in cytokine expression and secretion in response to nLDL and ssRNA40 treatments (Fig. $2 \mathrm{f}$ and $\mathbf{2 g}$ ). Thus, TLR8 is both necessary and sufficient for nLDL-induced activation of NF-KB in human macrophages.

The functionality of TLR8 in mice has been controversial, as murine TLR7 recognizes ligands of both human TLR7 and TLR8 and is required for detection of synthetic and viral SsRNA ${ }^{29,30}$. Additionally, mouse TLR8 lacks a 5-amino acid motif that is necessary for proteolytic processing, folding and activity of human TLR8 ${ }^{31,32}$. Nonetheless, canonical signaling domains required for activation of NF-kB remain highly conserved and are activated in the presence of synthetic thymidine homopolymer oligonucleotides ${ }^{33}$, suggesting the possibility of undiscovered natural ligands. To determine whether nLDL is capable of activating murine TLR7 and/or TLR8 we over-expressed each gene in HEK293T cells containing a NF-kB-driven luciferase reporter. Intriguingly, nLDL activation of NF-kB was specific for mouse TLR8, despite a lack of responsiveness to synthetic ssRNA and nucleoside analogues (Fig. $2 \mathrm{~h}$ ). Moreover, BMDM of wild-type and TIr7-deficient mice showed similar sensitivity to $\mathrm{nLDL}$, indicating TLR7 is not necessary for nLDL-induced macrophage activation in mice (Fig. 2i). nLDL activation of both mouse and human TLR8 was found to be dose-dependent in luciferase-reporter assays, but only human TLR8-activity was blocked by the chemical inhibitor CU-CPT9a (Fig. 2j). Collectively, these data support a conserved sensitivity of TLR8 to nLDL in both mice and humans.

\section{nLDL-msRNAs mediate inflammatory signaling in macrophages}

Given the innate immune function of TLR8 in detecting foreign ssRNA, nLDL may activate macrophages through RNA, rather than lipid, cargo. We have previously reported that apoB-containing lipoproteins in mice are enriched with microbial small RNAs (msRNA) that are likely derived from microbes of our internal and external environment ${ }^{34}$. To determine if human LDL also transports msRNAs, sRNAs were quantified on human nLDL by sRNA-seq (Fig. 3a). LDL-sRNA reads that aligned to the human reference genome were given priority, which revealed human sRNAs-derived from parent rRNAs (rDR) and tRNAs (tDR) to be most abundant, followed by microRNAs (Fig. 3b). Reads that failed to align to the human genome were then aligned concurrently to structural RNA databases (i.e. rRNA (SILVA) and tRNA (tRNA$\mathrm{db}$ )), as well as, curated genomes for bacteria and other microbes (Fig. 3c,d and Supplementary Fig. 2). On average, non-host sRNA were found to be approximately sixteen-times more abundant than host sRNAs, with bacterial rDRs explaining approximately $30 \%$ of all mapped reads (Fig. 3a). Alignment to a 
database of curated bacterial genomes found enrichment for msRNAs-derived from Proteobacteria, although one individual showed enrichment for Firmicutes msRNAs, and several showed high abundance of Actinobacteria msRNAs (Fig. 3d and Supplementary Fig. 2). In addition, alignment to curated databases for fungi and algae genomes also found substantial mapping relative to the host genome (Supplementary Fig. 2). To distinguish the inflammatory potential of endogenous and exogenous sRNAs on $\mathrm{nLDL}$, sRNAs, with comparable size to those found on LDL, were isolated from human cells (representing host sRNAs) and from E. coli (representing non-host msRNA)(Fig. 3e) and transfected in equal concentrations to THP-1 macrophages. Remarkably, host-derived sRNAs were unable to activate inflammatory signaling in human macrophages, whereas foreign msRNAs promoted the expression of IL 1B, IL6 and IL 12B, comparable to the synthetic ssRNA ORN06 (Fig. 3f). Lastly, to determine if sRNA cargo confer $n L D L ' s$ inflammatory capacity in macrophages, reconstituted LDL particles lacking sRNAs were generated by selectively removing the neutral-lipid core of $\mathrm{nLDL}$ and replacing it with cholesteryllinoleate spiked with a fluorescent cholesteryl ester ${ }^{35}$. Phospholipid-to-protein ratios were similar for rLDL and nLDL and exogenous lipid loading was confirmed by fluorescence (Supplementary Fig. 2). Although APOB levels were similar between $r L D L$ and $n L D L$, rLDL particles lacked sRNA cargo, as determined by bioanalyzer analysis (Fig. 3g) and qPCR (Fig. 3h and Supplementary Fig. 2). To determine if rLDL lacking sRNAs activate macrophages, THP-1 cells were treated with equal concentrations rLDL and nLDL. Although macrophages showed robust uptake of both rLDL and nLDL (Fig. 3i), only nLDL was associated with induction of inflammatory gene expression and cytokine secretion (Fig. 3j,k). These results support that the msRNA cargo confer nLDL's pro-inflammatory effects in macrophages.

\section{nt-LNA inhibits nLDL activation of macrophage TLR8}

Chemically modified (e.g. 2'-0-methyl and locked-nucleic acid (LNA)) microRNA (miRNA) inhibitors have been reported to impair sSRNA activation of TLR7/8 in macrophages and dendritic cells, likely independent of miRNA:mRNA targeting ${ }^{36-38}$. We hypothesized that dispensing with miRNA-targeting could yield a viable strategy for antagonizing TLR8 activation by LDL-msRNA. Hence, we screened commercially available non-targeting LNAs (nt-LNA; i.e. designed to avoid transcriptome-wide interactions) for TLR8 antagonism using NF-KB (luciferase) reporter assays (Fig. 4a; Supplementary Table 4). Based on NF-KB activity, nt-LNAs proved to be powerful inhibitors of TLR8-mediated responses to ssRNA ligands, but relatively poor inhibitors of nucleoside ligands. The strongest inhibitory effects were observed with nt-LNAs containing a phosphorothioate backbone (nuclease protection). Doseresponse curves support that nt-LNA likely functions as a competitive antagonist of human TLR8 for ssRNA ligands (Fig. 4b). Although both sequences were effective, the nt-LNA-A oligonucleotide, referred to herein as nt-LNA, was found to be a more effective antagonist. Furthermore, nt-LNA pre-treatments in THP-1 macrophages dose-dependently blocked activation of cells by ssRNA40, but not CLO75 and other TLR ligands (Supplementary Fig. 3).

To determine if nt-LNA inhibits nLDL activation of human macrophages, THP-1 cells were pre-treated with nt-LNA prior to and during nLDL treatments for $24 \mathrm{~h}$. Remarkably, nt-LNA were found to nullify nLDL activation of human macrophages based on the significant reduction of inflammatory gene (mRNA) 
expression (Fig. 4c). Concordantly, nt-LNA treatments also blunted nLDL-induced IL-6 and TNF-a secretion (Fig. 4d) and intracellular IL-1 $\beta$ and IKK $\beta$ protein levels (Fig. 4e). To further illustrate the mechanism of nt-LNA function in TLR8 signaling, primary BMDMs were transfected with nt-LNA in the presence of interferon gamma (IFN- $\gamma$ ), which has previously been demonstrated to promote proteosomebased turnover of IkBa, removing a negative feedback loop on NF-kB activity ${ }^{39}$. At the mRNA level, nLDLmediated inductions of $/ / 1 \mathrm{~b}$ and $/ / 6$ were significantly impaired by nt-LNA treatments (Fig. 4f). IL-6 secretion in response to $\mathrm{nLDL}$ treatments were also blocked by nt-LNA (Fig. $\mathbf{4 g}$ ). In a temporal experiment, $\mathrm{nLDL}$ were found to be rapidly internalized by BMDMs, as evidenced by increased intracellular APOB protein levels and promoted lipid-droplet formation, as assessed by PLIN2 protein levels, both of which were not impaired by nt-LNA treatments (Fig. 4h). nLDL treatment was associated with reduced IKK $\beta$ protein, likely due to enhanced proteosome activity, which was blocked by nt-LNA pre-treatment. As expected, IFN-y pre-treatment resulted in barely detectable levels of $\mathrm{lkBa}$, which were unexpectedly restored by nt-LNA pre-treatment and further stabilized in the presence of nLDL. Lastly, pre-treatment with nt-LNA promoted a rightward shift in nLDL-dose response curves of HEK293T reporter cells co-expressing mouse TLR7 and TLR8 (as is found endogenously in BMDM) or human TLR8 alone (as is found in human macrophages; Fig. 4i). Collectively, results support that nt-LNA is an effective competitive antagonist for LDL-msRNA-mediated activation of TLR8.

\section{nt-LNA inhibit atherosclerosis progression}

To determine if nt-LNA treatments reduce atherogenesis in vivo, adult female hypercholesterolemic $\left(A p o e^{-/}\right)$mice were placed on an atherogenic diet for four weeks and injected weekly with nt-LNA or saline control (Fig. 5a). Most importantly, nt-LNA treatments were found to reduce atherosclerosis in mice by approximately $33 \%$ compared to saline control in absence of altering plasma lipid levels and minor changes in plasma apoA-I and apoB (Fig. 5b-d and Supplementary Fig. 4). Neither collagen content (Masson's Trichrome; (Fig. 5e,f), nor macrophage numbers (F4/80 and Mac2) in aortic lesions were significantly altered by nt-LNA treatments (Fig. $\mathbf{5 g - j}$ ). Treatment with nt-LNA did not result in any changes to hepatic lipid metabolism or inflammatory gene expression, suggesting specificity for TLR8 antagonism in the lesion (Supplementary Fig. 4). To determine if nt-LNA promote lesion regression, adult LDL receptor-deficient $\left(L d r^{\prime /}\right)$ mice were fed an atherogenic diet for 14 weeks to develop severe atherosclerotic lesions and then switched to a chow control diet for an additional 4 weeks, as previously described ${ }^{40}$. After conversion to the chow diet, remaining mice were injected weekly with nt-LNA or saline (Fig. 5k). At baseline (14-weeks), mice on atherogenic diet showed expected elevations in plasma lipids compared to mice fed a chow diet, which returned to normal levels after 4 weeks of chow diet (Fig. $\mathbf{5 1 , m}$ and Supplementary Fig. 4). No significant differences in circulating apoA-I or apoB protein levels were observed between nt-LNA-treated and saline-treated mice after 4 weeks of treatments. Yet, nt-LNA treatments were found to significantly reduce atherosclerotic lesion area compared to control mice, which was more pronounced in males than females (Fig. 5n,o and Supplementary Fig. 4). Similar to the progression study, we failed to observe a significant change in fibrosis (Supplementary Fig. 4) or macrophage content between the regression treatment groups, though a trend towards increased Mac2 ${ }^{+}$ 
macrophages was observed in nt-LNA-treated mice (Fig. 5p,q). These results support that nt-LNA treatments reduce lesion progression and promote lesion regression in mouse models of atherosclerosis.

\section{nt-LNA reduces inflammatory macrophage phenotypes in atherosclerotic lesions.}

Based on in vitro observations that nt-LNA reduced $n L D L$-induced activation of macrophages and in vivo findings that nt-LNA treatments reduced atherosclerosis in the absence of reducing macrophage area in lesions, single-cell RNA sequencing (scRNA-seq) was performed to assess nt-LNA effects on lesion macrophage phenotypes. $\mathrm{CD} 45^{+} \mathrm{CD}^{-}$immune cells were isolated by fluorescence-activated cell sorting from aortic lesions of atherosclerotic mice (Apoe-- mice on atherogenic diet) administered with nt-LNA or a saline control for four weeks (Supplementary Figs. 5,6). In total, 2,742 and 4,871 quality cells (representing $92 \%$ of total input cells) were obtained from control and nt-LNA-treated lesions, respectively. This approach identified 20 distinct clusters that were broadly identified as myeloid cells (9/20), B-cells (4/20), T-cells (6/20) and natural killer (NK)-cells (1/20), based on gene enrichment analysis (Fig. 6a and

Supplementary Fig. 6; Supplementary Table 5). The number of total myeloid cell counts were comparable for both treatment groups; however, myeloid cells accounted for only a third of the cells detected in the ntLNA lesions, suggesting potential lymphocyte expansion with nt-LNA treatments (Fig. 6b). Nevertheless, differential gene expression analyses between treatment groups identified relatively few differences among lymphocytic groups (Supplementary Fig. 6 and Supplementary Table 6), whereas robust changes were detected in myeloid cells between treatments (Fig. 6c and Supplementary Table 7). Among genes suppressed by nt-LNA treatments in myeloid cells were critical inflammasome mediators $/ / 1 \mathrm{~b}$ and N/rp3, as well as regulators of foam cell formation (Cebpb and Ctsd) (Fig. 6d) ${ }^{41-43}$. Gene ontology analysis of all transcripts significantly altered by nt-LNA in predicted myeloid cells identified a significant enrichment of genes associated with immune responses, and more specifically leukocyte activation, supporting the hypothesis that nt-LNA's anti-atherosclerotic effects are due to reduced macrophage activation (Fig. $\mathbf{6 e}$ and Supplementary Table 8). To further investigate this point, myeloid cells were re-clustered to enhance phenotypic resolution (Fig. 6f,g). Based on enrichment of putative marker genes, specific macrophage phenotypes were identified, including inflammatory (C3), foamy (C6), cavity (C8), proliferating (C0), repair (C14), and two clusters of resident macrophages (C1 and C4) (Fig. 6f-i and Supplementary Table 9). Remarkably, inflammatory and foamy macrophages clusters were found to be predominant in lesions from saline-treated mice, whereas proliferating, cavity, and repair macrophages were more prevalent in lesions of nt-LNA-treated mice (Fig. 6g,i). Classical monocytes (C7), recently characterized aortic intimal resident macrophages (Mac-AIR) (C9), mixed monocyte/macrophage/dendritic cells (cDC2; C2), and a neutrophil-like cluster of cells (C11) were roughly equal between treatments, whereas mature (C12), plasmacytoid (C13) and CDC1 (C10) dendritic cells, as well as resident (C1/C4) and transitioning (C5) macrophages were skewed towards nt-LNA-treated mouse lesions. Repair macrophages (C14) were found to be approximately $80 \%$ derived from nt-LNA-treated mice and showed high expression of Adgre 1 (F4/80), thus supporting immunohistochemistry data (Fig. 5h). Collectively, results support that nt-LNA treatments promote the reorganization of macrophage phenotypes from pro-inflammatory and lipid- 
storage macrophages towards expansion of resident and repair macrophages that correlate with reduced disease burden.

\section{Discussion}

Our findings support that a diverse collection of exogenous SRNA are present on LDL, and upon uptake by macrophages, trigger inflammatory activation through TLR8 (Fig. 7). The mechanisms and route by which LDL acquire msRNA remains entirely unexplored; however, phagocytotic cells within mucosal tissues, which serve as the interface to the microbial environment, likely contribute to the acquisition and loading of msRNA on circulating LDL. We postulate that acquisition and transport of msRNA by LDL likely highlights a novel physiological role of lipoproteins that is at least partially independent of lipid trafficking and may serve to maintain mucosal homeostasis. Our results support a model in which impaired lipoprotein clearance and subsequent deposition of LDL within the vascular wall promotes nonsaturable uptake by macrophages, which in turn propagate vascular inflammation following recognition of LDL-msRNA cargo. Critical support of this model lies in our finding that removal of sRNA cargo from LDL depletes its inflammatory potential, as well as our observations that impeding the cellular responses to $\mathrm{nLDL}$-msRNA blunts macrophage activation in response to $\mathrm{nLDL}$ and reduces atherosclerotic burden in hypercholesterolemic mice. The identification of LDL-msRNA as inciting inflammatory factors overcomes a major barrier in our understanding of sterile inflammation that frequently accompanies metabolic diseases.

Further studies are needed to gain a greater understanding of the impact of genetic, environmental, lifestyle, and socio-economic factors on LDL-msRNA content, as such investigation may identify novel risk factors for human disease, particularly CVD. Although many TLR-modulating agents have been considered for combating CVD and other metabolic diseases, strategies to selectively antagonize TLR8 are underdeveloped ${ }^{44}$. The identification of nt-LNA as competitive antagonists of LDL-msRNA by TLR8 highlights a new avenue for therapeutic development, one that is likely to be well-tolerated in human subjects ${ }^{45}$. The development of therapeutic strategies to mitigate LDL-msRNA signaling may hold great potential for treatment and prevention of CVD, as well as many other diseases that feature hyperlipidemia and chronic inflammation.

\section{Online Methods}

\section{Materials}

Coding sequences of individual toll-like receptors (TLR) were amplified from cDNA of human and mouse macrophages using Phusion high-fidelity DNA polymerase (ThermoFisher) and cloned to the multiplecloning site of the pAdTrack-CMV vector, which expresses GFP from an independent CMV promoter (Addgene). The coding sequence of the TLR chaperone protein UNC93B1 and co-receptor CD14 were similarly amplified from human macrophage cDNA and inserted to the pMSCV-neo and pMSCV-puro vectors, respectively (Clontech). The NanoLuc Reporter Vector with NF-kB Response Element (pNL3.2.NF- 
kB-RE) is commercially available (Promega). Transfections of plasmid DNA were performed using Lipofectamine 3000 (LifeTechnologies), per manufacturer's instructions. In vivo grade nt-LNA (Cat\# 339203, Qiagen) was purchased in individual lots of $100 \mathrm{mg}$, reconstituted in sterile saline, aliquoted, and stored at -20C. Single-stranded RNA oligonucleotides were synthesized with a phosphorothioate backbone (PS; Integrated DNA Technologies). Nucleoside analogues CL075 and Resiquimod (R848) were purchased commercially (Sigma). Bafilomycin A1 and EGTA-AM were purchased commercially (Sigma). For transfections, nt-LNA, ssRNA, and sRNA oligonucleotides were complexed with DOTAP (Sigma) according to manufacturer's instructions. Ligands for TLR1/2 (Pam3CSK4), TLR2 (Heat-killed Listeria monocytogenes), TLR3 (Poly(I:C)), TLR4 (LPS Escherichia coli K12), TLR5 (Flagellin Salmonella typhimurium), TLR6/2 (FSL-1) and TLR9 (ODN2006) were purchased commercially (Invivogen) and diluted according to manufacturer's recommendations. Antibodies used for immunoblotting and flow cytometry in this study are provided in Supplementary Table 10.

\section{Mouse studies}

Animals and models of atherosclerosis

Wild-type (WT), $T / r T^{/-}, L d l r^{--}$and $A p o e^{-/-}$mice of the C57BL/6 background were purchased (The Jackson Laboratory). Animal protocols were approved by and performed according to the regulations of Vanderbilt University Medical Center's Institutional Animal Care and Usage Committee (IACUC) and animal studies complied with all relevant ethical regulations for vertebrate animal research (\#M1600252$01)$. Mice were maintained on a chow diet $(\mathrm{NIH}-31)$ or an atherogenic diet containing $42 \% \mathrm{kcal}$ from milk fat and $0.2 \%$ cholesterol added (Envigo; TD.88137) in a 12h:12h light-dark cycle with unrestricted access to food and water. For atherosclerosis progression studies, 10-week-old female Apoe-/- mice were converted from a chow diet to a atherogenic diet for 4 weeks receiving weekly intraperitoneal injections of saline $(n=10)$ or non-targeting locked-nucleic acid ( $n t-L N A ; 30 \mathrm{mg} / \mathrm{kg} ; \mathrm{n}=10)$ that were randomized within cages to limit cage effects. For atherosclerotic regression studies, male and female $L d I^{-/-}$mice were fed the atherogenic diet for 14-weeks to promote hypercholesterolemia and lesion development ( $n=25 / \mathrm{sex}$ ). $L d l r^{-/-}$mice fed a chow diet for this time period were used as an age-matched control. After 14-weeks of atherogenic diet feeding, mice from each cage were randomly assigned to either a baseline group that was sacrificed at this time ( $n=7-8 /$ sex), or one of two treatment groups that were converted to a chow diet for 4 additional weeks with weekly intraperitoneal injections of saline ( $n=9 / \operatorname{sex})$ or $n t-L N A(n=8-9 / \operatorname{sex})$, as previously described ${ }^{40}$. Results of the regression study are pooled from two independent cohorts of mice staggered by 4 weeks. At sacrifice, blood was collected by cannulating the vena cava using an EDTAcoated syringe and plasma was obtained by centrifugation at 2,500 $\mathrm{xg}$ for $10 \mathrm{~min}$. Isolated plasma was stored at $-80^{\circ} \mathrm{C}$ prior to analysis. Unless otherwise indicated, tissues were flash frozen in liquid nitrogen and stored at $-80^{\circ} \mathrm{C}$.

Lipid analysis of mouse plasma 
Plasma collected during animal sacrifice was assayed directly for total cholesterol and triglycerides using colorimetric kits (Pointe Scientific). For assessment of lipid/lipoprotein distribution, $200 \mu \mathrm{L}$ of mouse plasma was diluted in SEC running buffer and injected in the ÄKTA SEC system composed of three tandem Superdex-200 Increase columns with collection of $1.5 \mathrm{~mL}$ fractions. Individual fractions were then assessed for total protein (BCA; Pierce) and lipids (Pointe Scientific).

\section{Quantification of Atherosclerosis:}

For each experiment, mice were fasted for $4 \mathrm{~h}$ at the end of the dark phase and sacrificed with isoflurane. Blood was collected by cannulating the vena cava and mice were perfused with 1X PBS until vasculature was clear. The heart, with aortic root attached was harvested, embedded with OCT and frozen on dry-ice. As detailed by Paigen et al. ${ }^{46}, 30,10 \mu \mathrm{m}$ sections were taken from the beginning of the aortic root (where valve-cusps first appear) using a Leica CM3050s cryostat by the same technician for all studies. For each root, every other slide for the 300- $\mu \mathrm{m}$ span was stained with Oil-Red-O (ORO) to identify the neutral lipid deposition. To quantify lesion area, ORO positive area was imaged using the KS300 imaging system (Kontron Elektronik $\mathrm{GmbH}$ ), as previously described ${ }^{47-49}$, and reported as $\mathrm{ORO} / \mathrm{mm}^{2}$. The area under the curve (AUC) was calculated from the lesion area of serial sections. All other stains were conducted on sections 40-60 mm distal of the aortic sinus. Masson's Trichrome staining and immunohistochemistry for F4/80 (Novus Biologicals; secondary by Vector Laboratories) were performed by the Vanderbilt Translational Pathology Shared Resource core using 4 sections per animal and quantified using the KS300 imaging system applying methodologies previously described ${ }^{50}$. For immunofluorescence studies, frozen aortic root sections were dried at $55^{\circ} \mathrm{C}$ for 10 minutes and then fixed in formalin for 20 minutes. Slides then underwent $3 \times 30$ dip rinses in deionized water. Slides then underwent antigen retrieval $(\mathrm{H}-$ 3300 , VectorLabs) in a pressure cooker for 10 seconds. Slides were then rinsed (30 dips) in 1x PBS 3 times. In a humidified chamber, slides were blocked using 1\% BSA for 30 minutes and then aspirated and incubated overnight in primary antibody (Mac2, 1:10,000, Cedarlane). Slides were then rinsed (30 dips) 3X in 1X PBS and then probed with secondary antibody (Goat anti-rat AF488, 1:200, ThermoFisher) and sealed with a coverslip. Fluorescence microscopy was completed using a Nikon STORM scope and images were recorded for subsequent analysis in Image-J (Fiji).

\section{Cell culture studies}

\section{Tissue Culture:}

HEK293T cells were maintained in DMEM supplemented with sodium pyruvate ( $110 \mathrm{mg} / \mathrm{L}), \mathrm{L}$-glutamine, $10 \%$ heat-inactivated FBS, and $1 \%$ penicillin-streptamycin (GIBCO). THP-1 cells were maintained in RPMI1640 supplemented with 25 mM HEPES, L-glutamine, 10\% heat-inactivated FBS, and 1\% penicillinstreptamycin (GIBCO) on suspension culture dishes (Corning). To generate THP-1 macrophages, THP-1 cells were suspended in fresh media spiked with $5 \mathrm{ng} / \mathrm{mL}$ 12-myristate 13-acetate (PMA) and transferred to non-tissue culture treated plates for $48 \mathrm{~h}$. After $48 \mathrm{~h}$, adherent cells were washed twice with PBS and given fresh media without PMA for $24 h^{51}$. For siRNA experiments, non-differentiated THP-1 cells were 
concentrated 10-fold in Opti-MEM and incubated with $300 \mathrm{mM}$ scrambled or siTLR8 siRNA (ON TARGETplus; Horizon Discovery), or without siRNA, and electroporated using program Y-001 on a Nucleofector $2 \mathrm{~b}$ device (Lonza). Cells were immediately diluted 10 -fold in RPMI containing $5 \mathrm{ng} / \mathrm{mL}$ PMA and differentiated, as above. Primary bone marrow-derived macrophages (BMDM) were generated by incubating total bone marrow aspirates isolated from the tibia and femur of C57BL/ 6 mice in DMEM containing sodium pyruvate $(110 \mathrm{mg} / \mathrm{L})$, L-glutamine, $10 \%$ heat-inactivated $\mathrm{FBS}$, and $1 \%$ penicillinstreptamycin (GIBCO) supplemented with murine GM-CSF ( $50 \mathrm{ng} / \mathrm{mL}$; Tonbo Bioscience) for $72 \mathrm{~h}$ on nontreated tissue culture plates ${ }^{52,53}$. After $72 \mathrm{~h}$, non-adherent cells were washed with PBS and provided additional fresh media containing GM-CSF with indicated treatments.

NF-KB Activity (Luciferase) Reporter Assay:

HEK293T cells were seeded in 24- or 96-well plates and allowed to attach overnight in full media. The following day, cells were transfected with $1 \mathrm{mg} / \mathrm{mL}$ plasmid DNA (35\% pAdTrack-CMV, 30\% pMSCV-puroCD14, 30\% pMSCV-neo-UNC93B1, 5\% pNL3.2.NF-kB-RE) using Lipofectamine 3000. After 3h, transfection media was carefully aspirated and replaced with fresh media for $24 \mathrm{~h}$. The following day treatments were performed as indicated for 20-24h at which time media was removed and a passive lysis buffer (NanoGlo Luciferase Assay Buffer; Promega) was added, and lysates were incubated on a horizontal shaker for 10 min at $25^{\circ} \mathrm{C}$ with $500 \mathrm{RPM}$. Lysates were then transferred to an opaque 96-well plate for measurement of GFP (co-expressed with GOI from pAdTrack-CMV) fluorescence as an indicator of cell density and transfection efficiency on a Synergy Mx plate-reader. Subsequently, the NanoLuc substrate Furimazine was diluted in lysis buffer, added to lysates with a multi-channel pipette, and incubated with agitation for 2 min at room temperature. Luminescence was then quantified using a Synergy Mx plate-reader. Luciferase activity was calculated as luminescence divided by fluorescence and reported as a relative fold change.

\section{Methods}

RNA Isolation and Real-time PCR:

For cells, total RNA was isolated using Total RNA Purification kits (Norgen), as per manufacturer's instructions. For tissue, total RNA was isolated using miRNeasy Mini kits (Qiagen), as per manufacturer's instructions. For lipoproteins, total RNA was isolated using miRNeasy Mini kits with slight modifications to the manufacturer's instructions. Briefly, Qiazol was added at 10x initial volume of lipoprotein concentrate, vortexed for 30-60 s and allowed to incubate for 5 minat room temperature prior to addition of 0.2 volumes of chloroform. After phase separation, the aqueous phase was mixed with an equal volume of $100 \%$ ethanol, vortexed, and stored overnight at $-80^{\circ} \mathrm{C}$. The following day, the RNA sample was applied to the mini column per manufacturer's instructions. For targeted isolation of human small RNAs, $1 \times 10^{7}$ THP-1 cells were pelleted by centrifugation and lysed in Qiazol and processed according to Appendix A of manufacturer's instructions (Qiagen). For targeted isolation of bacterial sRNA, an overnight culture of $E$. coli (DH5a; Invitrogen, Cat\# 18265-017) was pelleted at 4,000 x g, washed $2 \mathrm{X}$ with $\mathrm{PBS}$, and 
incubated with $1 \mu \mathrm{g} / \mathrm{mL}$ lysozyme for $5 \mathrm{~min}$ at room temperature with agitation. Qiazol was then added and the partial cell lysate was pulverized with Zirconium beads (Sigma) in an oscillating tissue homogenizer for $30 \mathrm{~s}$. Subsequently, the sample was processed following the Appendix A protocol of the miRNeasy Mini kit (Qiagen) for the separate isolation of long and small RNAs. Total RNA, sRNA and LDLsRNA were assessed by PicoChip on a 2100 Bioanalyzer instrument (Agilent). cDNA was generated from total RNA using the High Capacity cDNA Reverse Transcription Kit (ThermoFisher) for mRNA targets. For sRNA targets, the miRCURY LNA RT kit was used for cDNA-synthesis (Qiagen). Real-time PCR was performed for targeted transcripts and sRNAs using the Power SYBR 2X Master Mix (ThermoFisher) on QuantStudio 6 or QuantStudio 12 instruments (LifeTechnologies). RPOL// served as a housekeeper for human cells and Rplp01 (36b4) served as a housekeeper for murine cells and tissues For LDL-sRNAs, an arbitrary $\mathrm{Ct}$ of 35 was used for RQV calculations. All primer sequences are available upon request. Relative quantitative values (RQV) were calculated by the delta-Ct method and reported as fold changes.

\section{High-throughput RNA Sequencing:}

Individual total RNA sequencing libraries were generated using Ovation Human FFPE RNA-Seq Multiplex System (NuGen) kits, per manufacturer's instructions. Individual libraries were assessed for quality using the Agilent 2100 Bioanalyzer and quantified with a Qubit Fluorometer. Paired-end sequencing of multiplexed libraries was performed on the NovaSeq6000 (Illumina) platform by the Vanderbilt Technologies for Advanced Genomics (VANTAGE) core (Vanderbilt University, Nashville, TN). Reads were trimmed to remove adapter sequences using Cutadapt v1.16 ${ }^{54}$ and aligned to the human hg19 genome using STAR v2.5.3a ${ }^{55}$. GENCODE human v19 gene annotations were provided to STAR to improve the accuracy of mapping. Quality control on both raw reads and adaptor-trimmed reads was performed using FastQC (www.bioinformatics.babraham.ac.uk/projects/fastqc). featureCounts v1.15.2 ${ }^{56}$ was used to count the number of mapped reads to each gene. Significantly differential expressed genes with FDRadjusted $p$-value $<0.05$ and absolute fold change $>2.0$ were detected by DESeq2 (v1.18.1) ${ }^{57}$. Gene ontology and transcription factor analyses were assessed from differentially expressed mRNAs using the MetaCore software suite (Clarivate Analytics).

\section{Small RNA Sequencing:}

Individual small RNA libraries were generated from LDL-sRNA using the NEXTFlex Small RNA Library Preparation Kits v3 for Illumina Platforms (Perkin-Elmer), per manufacturer's instructions with the following modifications: 1) 3'- and 5'-adapters were diluted 1:8, 2) 3'-adapter ligation was performed overnight in multiple steps $-25^{\circ} \mathrm{C}$ for $2 \mathrm{~h}, 20^{\circ} \mathrm{C}$ for $4 \mathrm{~h}$ and $16^{\circ} \mathrm{C}$ overnight, 3 ) following cDNA synthesis and prior to barcoding PCR, the supplementary modified step F protocol was followed (i.e. no size selection protocol), 4) PCR amplification was 20 cycles. Following PCR amplification, individual libraries were size-selected (136-200 bp product) using pre-cast $3 \%$ agarose gel cassettes with a Pippin Prep (Sage Sciences) and quantified on a Qubit Fluorometer. Single-end sequencing (SE-75) of equimolar multiplexed libraries was performed on the NextSeq500 (Illumina) platform by the Vanderbilt Technologies for Advanced Genomics (VANTAGE) core (Vanderbilt University, Nashville, TN). 
Bioinformatic processing was performed using in-house data analysis pipeline (TIGER), previously described in detail ${ }^{34}$. Briefly, Cutadapt ${ }^{54}$ was used to trim $3^{\prime}$ adapters for raw reads. All reads with less than 16 nucleotides (nts) were designated as "too short" and discarded. The adaptor-trimmed reads were mapped to the hg19 genome, with additional rRNA and tRNA reference sequences, by Bowtie1 (v1.1.2) ${ }^{58}$ allowing only one mismatch $(1 \mathrm{MM})$. Reads $<20 \mathrm{nts}$ that failed to be annotated as sRNA without perfect alignment (PM) to human genome were discarded. Remaining unmapped reads were then mapped in parallel to exogenous structural RNA databases and curated microbial genome databases allowing no mismatches (PM). Reads that failed to align to any strategy were categorized as "unknown".

\section{Single Cell RNA Sequencing:}

After collection of the heart and aortic root, as described above ( $n=8 /$ treatment), the aortic arch and thoracic aorta were removed, cleaned, and placed into $1 \mathrm{~mL}$ of RPMI 1640 containing $10 \%$ FBS on ice, as previously described ${ }^{59}$. Once all aortas were harvested, aortas were minced to small pieces using vigorous scissor action and digested by shaking at $37^{\circ} \mathrm{C}$ for $30 \mathrm{~min}$ in $1 \mathrm{~mL}$ of RPMI 1640 containing 2.6 $\mathrm{U} / \mathrm{mL}$ Liberase TM (Sigma), $60 \mathrm{U} / \mathrm{mL}$ hyaluronidase (Sigma), $0.1 \mathrm{mg} / \mathrm{ml}$ DNase I (Sigma), $1 \mu \mathrm{g} / \mathrm{mL}$ actinomycin D (Sigma), $0.744 \mathrm{U} / \mathrm{mL}$ Elastase (Worthington Biochemical). Digested Samples were placed in fresh tubes and spun at $500 \mathrm{xg}$ for $10 \mathrm{~min}$ at $4^{\circ} \mathrm{C}$. Pellets were pooled and filtered through a $70 \mathrm{~mm}$ cell strainer using RPMI + 10\% FBS. Fc receptors were blocked with anti-mouse CD16/32 (BioLegend) for 10 min at $4^{\circ} \mathrm{C}$ prior to surface marker staining. Cells were stained with Pacific Blue anti-CD 45 (BioLegend); FITC anti-Ter119/RBC (BD Biosciences); and PerCP-Cy5.5 anti-CD3 (BD Pharmingen) for 30 min at $4^{\circ} \mathrm{C}$. Cells were washed $2 \mathrm{X}$ with RPMI $+10 \% \mathrm{FBS}$, filtered, and sorted using a 4-laser FACSAria III by the VUMC Flow Cytometry Shared Research Core. Dead cells and red blood cells were excluded and $C D 45^{+} \mathrm{CD} 3^{-}$cells were collected using a 100 mm nozzle into RPMI $1640+10 \%$ FBS. Each sample (saline $=13,055$ cells; ntLNA $=19,980$ cells) was processed for single cell 3' RNA sequencing utilizing the $10 X$ Chromium system through the Vanderbilt Technologies for Advanced Genomics (VANTAGE) core (Vanderbilt University, Nashville, TN). Libraries were prepared using P/N 1000006, 1000080, and 1000020 following the manufacturer's protocol. The libraries were sequenced using the NovaSeq6000 with 150 bp paired-end reads (PE-150). RTA (version 2.4.11; Illumina) was used for base calling and analysis was completed using 10X Genomics Cell Ranger software v2.1.1. scRNABatchQC software package was used for assessment of scSEQ data quality control, as previously described ${ }^{60}$. Clustering analysis was generated by Seurat ${ }^{61}$. Initial cell type classification (i.e. myeloid/macrophage, B cells, T cells, NK cells) of complete datasets was based on cell activity database ${ }^{62}$. Refined annotation of clusters was conducted manually following recommendations of a meta-analysis of similar sequencing approaches ${ }^{17}$; however, discrepancies between predicted clusters based on the previously reported meta-analysis and our data were observed. These differences were likely attributable to the early atherosclerosis model employed in our study. Hence, we observed two resident macrophage clusters distinguishable by the expression of Bcell markers Cd79a, Ms4a1, Fcamrand Ighm (C1 vs C4), which may be the result of pre/pro-B cell differentiation to macrophages ${ }^{63}$. Aortic intimal resident macrophages (Mac-AIR; C9) were categorized by 
their expression of $C \times 3 c r 1$ and Itgax, as well as genes in lipid metabolism (Abcg1, Scarb1, Pparg, $C e b p b)^{64}$. Interestingly, this population also had selective expression of $C d 300$, as well as enriched expression of $\mathrm{Nr} 4 \mathrm{a} 1$, a transcription factor associated with resident macrophage differentiation ${ }^{65}$. Repair macrophages (C14) were categorized by their expression of Mlxip/ (ChREBP), Alox15 and Fn1 (Fibronectin) ${ }^{66}$. Transitioning cells (C5) showed expression of proliferation genes (Top2a, Mki67, Pclaf), as well as differentiation modulators Celf1 and Nr4a1. EdgeR software was used for differential expression analysis between treatments and within cell-types ${ }^{67}$. Gene ontology and transcription factor analyses were assessed from differentially expressed mRNAs using the MetaCore software suite (Clarivate Analytics).

\section{Lipoprotein Isolation and Preparation of Reconstituted LDL:}

Human whole blood was collected from fasted male and female subjects age 18 or older (IRB\#170046) in $\mathrm{K}_{2}$ EDTA tubes after informed written consent was obtained. Aliquoted plasma was either used fresh or stored at $-80^{\circ} \mathrm{C}$ and used after one freeze-thaw. Sequential density-gradient ultracentrifugation (DGUC) using a Beckman-Coulter Optima XPN-80 Ultracentrifuge with either SW32Ti or SW41Ti swinging bucket rotors was used to isolate VLDL (1.006-1.018), LDL (1.019-1.063), and HDL (1.064-1.021) by adjusting sample density with potassium bromide, as previously described ${ }^{68}$. Isolated lipoproteins were immediately dialyzed in 1 X PBS for at least 4 changes of 200 -fold excess buffer. After (up to) $48 \mathrm{~h}$ dialysis, lipoproteins were concentrated using Amicon Ultra Centrifugal Filters ( $3 \mathrm{kDa}$ molecular weight cutoff; Millipore) and then immediately sterile-filtered $(0.22 \mu \mathrm{m})$ by syringe. Total protein concentration was measured by BCA Protein Assay Kit (Pierce). Isolated lipoproteins were stored in the dark at $4^{\circ} \mathrm{C}$ and monitored for aggregation and loss of color. Best results were obtained from freshly collected plasma and newly processed $\mathrm{nLDL}$. As quality control, LDL isolated and stored under these conditions for up to one month were assayed for oxidation using the TBARs method, and compared against matched LDL oxidized by indicated concentrations and exposure time to copper sulfate ${ }^{69}$. Reconstituted LDL (rLDL) was prepared as previously described with minimal modifications ${ }^{35}$. Briefly, de-salted nLDL was mixed with potato starch (Sigma) by vortexing and flash frozen in liquid nitrogen prior to lyophilization for $6 \mathrm{~h}$. Neutral lipids were selectively removed by sequential heptane extractions at $-10^{\circ} \mathrm{C}$, prior to reconstitution with cholesteryl linoleate (NuChek-Prep) spiked with a 1:20 ratio of fluorescent 18:2 TopFluor cholesteryl ester (Avanti Polar Lipids) solubilized in heptane. This mixture was evaporated under a nitrogen stream until powder dry. Formation of rLDL particles was solubilizated in $10 \mathrm{mM}$ tricine, $\mathrm{pH} 8.4$ for $108 \mathrm{~h}$ at $4^{\circ} \mathrm{C}$. Insoluble starch was pelleted by centrifugation at $2000 \times \mathrm{g}$ and solubilized rLDL was extracted and sterilefiltered. In parallel, nLDL and rLDL samples were analyzed by size-exclusion chromatography (SEC) in running buffer $\left(10 \mathrm{mM}\right.$ Tris- $\left.\mathrm{HCl}, 0.15 \mathrm{M} \mathrm{NaCl}, 0.2 \% \mathrm{NaN}_{3}\right)$ using two tandem Superose- 6 gel filtration columns (Cytiva) on an ÄKTA SEC system (Cytiva). The distribution of fluorescent cholesteryl ester was measured by fluorometer using the Synergy Mx plate-reader. Total protein and lipid composition of LDL samples were determined by colorimetric kits: total cholesterol (Pointe Scientific), triglycerides (Pointe Scientific), Phospholipid C (Wako) and protein by BCA (Pierce). 
Immunoblotting:

Cell lysates were collected on ice in RIPA buffer $(150 \mathrm{mM} \mathrm{NaCl}, 1 \% \mathrm{NP}-40,0.5 \%$ sodium deoxycholate, $0.1 \%$ SDS, 50 mM Tris-HCl pH 8.0) containing Halt Protease and Phosphatase Inhibitors Cocktail and rotated at $4^{\circ} \mathrm{C}$ for $1 \mathrm{~h}$. Insoluble cell debris was pelleted at $12,000 \mathrm{xg}$ for $10 \mathrm{~min}$ in a pre-chilled centrifuge $\left(4^{\circ} \mathrm{C}\right)$, and soluble protein lysates were transferred to new tubes on ice. For immunoblots of cells, $12 \mu \mathrm{L}$ of cell lysate $(180 \mu \mathrm{L} /$ well of a 6-well plate) was mixed with $4 \mu \mathrm{L}$ of $4 x$ Protein Sample Loading Buffer (LICOR), denatured at $70^{\circ} \mathrm{C}$ for 5 minutes or $48^{\circ} \mathrm{C}$ for 10 min and immediately placed on ice. For immunoblots of mouse plasma, $1.5 \mathrm{~mL}$ of plasma was mixed with $10.5 \mathrm{~mL}$ RIPA buffer containing Halt Protease Inhibitors Cocktail (ThermoFisher) and $4 \mathrm{~mL}$ of $4 X$ Protein Sample Loading Buffer (LI-COR), denatured at $70^{\circ} \mathrm{C}$ for $5 \mathrm{~min}$ and immediately placed on ice. Denatured protein samples were then run on NuPAGE 4-12\% Bis-Tris or 3-8\% Tris-Acetate gels (Life Technologies) at 125V on ice with Precision Plus Protein Kaleidoscope Pre-Stained Protein Standards (BioRad) or HiMark Pre-Stained Protein Standards (Life Technologies) as molecular weight references. After electrophoresis, gels were transferred to Nitrocellulose membranes using the iBlot system (Life Technologies). Blots were then blocked in Intercept TBS Blocking Buffer (LI-COR) for 30 min prior to dilution of primary antibody. Primary antibodies were incubated for at least $1 \mathrm{~h}$ at room temperature or overnight at $4^{\circ} \mathrm{C}$, washed $3 \mathrm{X}$ with tris-buffered saline containing $0.05 \%$ Tween-10 for $10 \mathrm{~min}$. Subsequently, this process was repeated for secondary antibodies. Blots were assayed using the LI-COR Odyssey Infrared Imaging system, analyzed using Image Studio Lite and Image J software suites.

Single-plex and Multiplex ELISA:

Media supernatants of cell culture experiments were assayed for indicated cytokine concentrations by MILLIPLEX MAP Human Cytokine/Chemokine Magnetic Bead Panel (Millipore) on the Luminex MAGPIX System by the Vanderbilt Hormone Assay and Analytical Services Core, per manufacturer's recommendations. For individual assays, media cytokine concentrations were assayed by conventional ELISA for human and mouse IL-6 and TNF-a (DuoSet; R\&D Systems).

\section{Formulation of PLGA-CU-CPT9a microparticles:}

The selective TLR8 inhibitor CU-CPT9a is not water soluble, requiring alternative methods for effective drug delivery in cell culture systems. Hence, drug-loaded microparticles (MPs) were formulated using a conventional oil-in-water (O/W) emulsion technique. Poly(lactide-co-glycolide) (PLGA, 50:50 lactide: glycolide, $10 \mathrm{kDa}$, Sigma) was dissolved at $100 \mathrm{mg} / \mathrm{ml}$ in dichloromethane (DCM) with vortexing and brief sonication. CU-CPT9a (Sigma) was resuspended at $10 \mathrm{mg} / \mathrm{ml}$ in DCM. $0.5 \mathrm{~mL}$ of drug solution was added to $0.5 \mathrm{~mL}$ PLGA solution, at which point the drug fully dissolved. The polymer/drug 0 phase was added dropwise to $5 \mathrm{~mL} 1.5 \%$ poly(vinyl alcohol) (PVA, 30-70 kDa, Sigma) in water and homogenized for $30 \mathrm{~s}$ at 10,000 rpm with an IKA T18 Digital Ultra-Turrax homogenizer. The O/W emulsion was transferred to a $100 \mathrm{~mL}$ round bottom flask, and DCM was removed via rotary evaporation to complete MP hardening. The aqueous MP suspension was centrifuged at 7,500xg for $10 \mathrm{~min}$ and resuspended in 
deionized water to remove excess PVA, and the resuspended MPs were aliquoted for lyophilization and subsequently stored at $-20^{\circ} \mathrm{C}$. Immediately prior to experiments, lyophilized MP were equilibrated to room temperature and re-suspended with PBS to $4 \mathrm{mg} / \mathrm{ml}$ prior to dilution at indicated doses. Resulting particles were deposited on a glass coverslip, imaged on a Nikon Eclipse Ti inverted scope and diameters measured using Nikon Ti software. Drug loading was quantified by dissolving in dimethylsulfoxide and measuring the ultraviolet-visible absorbance spectra relative to free CU-CPT9a on a Varian UV-VIS spectrophotometer. Resulting particles were 3.39 +/- 1.93 microns in diameter with 84.8 microgram drug per mg MP (encapsulation efficiency $=84.8 \%$ ).

Statistics, Reproducibility and Rigor:

Data shown for in vitro experiments are representative experiments performed at least three times with independent donors/isolations of LDL. In vitro experiments were performed with technical replicates of 34. Unless otherwise specified data are mean $+/$ - SEM. For animal studies, all treatments were randomized within individual cages to limit cage effects. For atherosclerosis studies, quantification of lesion area, fibrosis, immunohistochemistry and immunofluorescence were assessed in a blinded fashion by at least two researchers. Harvest and preparation of heart and aortas for histology was also performed blinded by a single technician. Where two treatments were compared with a sample size $>3$ for each treatment a Mann-Whitney test (two-sided) was performed with alpha $=0.05$. Where three or more groups were compared a One-Way ANOVA was performed using Benjamini, Krieger and Yekutieli post-hoc test to control the false discovery rate $(\mathrm{Q}=0.05$; Fig. 1 and Fig. 4$)$ or Sidak's post-hoc test to control Type I errors (Fig. 5). Where more than one test was performed for three or more groups a Two-Way ANOVA was performed using Benjamini, Krieger and Yekutieli post-hoc test to control the false discovery rate $(Q=0.05)$. To determine whether differences existed between treatments in dose-response curves a nonlinear regression with extra sum-of-squares $\mathrm{F}$ test was performed. Schematics and aspects of some figures were created with BioRender.com, Tableau and R Studio. GraphPad Prism 8 (GraphPad Software) was used to create graphs and calculate statistical significances. Images were assembled in Affinity Designer (Serif) to generate figures.

\section{Declarations}

ACKNOWLEDGMENTS: The authors would like to thank Walter Reichard, Meaghan Kuzmich, Stuart Landstreet, Anca Ifram, Dr. Leslie Sedgeman, Dr. Carrie Wiese, and Dr. Vladimir Babaev for technical assistance and helpful discussions. We would also like to thank Dr. Qi Liu of the Vanderbilt Center for Quantitative Sciences for consultation on single-cell sequencing analysis, Angela Jones of VANTAGE at VUMC for expertise in high-throughput sequencing technologies, the Vanderbilt Flow Cytometry Shared Resource and Translational Pathology Shared Resource. This work is supported by American Heart Association awards 19CDA34660280 (R.M.A.) and 18IPA34180005 (R.M.A.), W.M. Keck Research Foundation Grant (R.M.A., M.F.L., Q.S., K.C.V.) and National Institutes of Health Grants P01HL116263 (M.F.L.), R01HL128996 (K.C.V.). 
AUTHOR CONTRIBUTIONS: R.M.A. Conceptualization, Methodology, Investigation, Formal Analysis, Visualization, Writing - Original Draft. D.L.M. Methodology, Investigation, Formal Analysis, Writing Reviewing and Editing A.B.C. Investigation, Formal Analysis. N.M. Investigation, Formal Analysis. E.M.S. Formal Analysis, Investigation. D.M.C. Resources, Formal Analysis. W.Z. Resources. C.J. Resources. M.C. Resources. Y.Z. Resources. C.A.R. Formal Analysis. M.R.S. Software, Visualization. S.Z. Software, Visualization. C.D. Methodology. A.C.D. Methodology, Writing - Reviewing and Editing. Q.S. Methodology, Software, Visualization, Writing - Reviewing and Editing. M.F.L. Methodology, Supervision, Writing Reviewing and Editing. K.C.V. Conceptualization, Methodology, Supervision, Formal Analysis, Visualization, Writing - Reviewing and Editing.

COMPETING INTERESTS: M.F.L. has received research funding from Amgen, Regeneron, lonis, Merck, REGENXBIO, Sanofi and Novartis and has served as a consultant for Esperion, Alexion Pharmaceuticals and REGENXBIO. All other authors have no competing interests.

\section{References}

1. Danesh, J., Collins, R., Appleby, P. \& Peto, R. Association of fibrinogen, C-reactive protein, albumin, or leukocyte count with coronary heart disease: meta-analyses of prospective studies. JAMA 279, 1477-1482 (1998).

2. Ridker, P. M., Rifai, N., Rose, L., Buring, J. E. \& Cook, N. R. Comparison of C-reactive protein and lowdensity lipoprotein cholesterol levels in the prediction of first cardiovascular events. N. Engl. J. Med. 347, 1557-1565 (2002).

3. Sabatine, M. S. et al. Evolocumab and Clinical Outcomes in Patients with Cardiovascular Disease. N. Engl. J. Med. 376, 1713-1722 (2017).

4. Ridker, P. M. et al. Antiinflammatory Therapy with Canakinumab for Atherosclerotic Disease. N. Engl. J. Med. 377, 1119-1131 (2017).

5. Moore, K. J. \& Tabas, I. Macrophages in the pathogenesis of atherosclerosis. Cel/ 145, 341-355 (2011).

6. Moore, K. J. \& Freeman, M. W. Scavenger receptors in atherosclerosis: beyond lipid uptake. Arterioscler. Thromb. Vasc. Biol. 26, 1702-1711 (2006).

7. Babaev, V. R. et al. Reduced atherosclerotic lesions in mice deficient for total or macrophage-specific expression of scavenger receptor-A. Arterioscler. Thromb. Vasc. Biol. 20, 2593-2599 (2000).

8. Suzuki, H. et al. A role for macrophage scavenger receptors in atherosclerosis and susceptibility to infection. Nature 386, 292-296 (1997).

9. Que, X. et al. Oxidized phospholipids are proinflammatory and proatherogenic in hypercholesterolaemic mice. Nature 558, 301-306 (2018).

10. Febbraio, M. et al. Targeted disruption of the class B scavenger receptor CD36 protects against atherosclerotic lesion development in mice. J. Clin. Invest. 105, 1049-1056 (2000). 
11. Stewart, C. R. et al. CD36 ligands promote sterile inflammation through assembly of a Toll-like receptor 4 and 6 heterodimer. Nat. Immunol. 11, 155-161 (2010).

12. Miller, Y. I. Toll-like receptors and atherosclerosis: oxidized LDL as an endogenous Toll-like receptor ligand. Future Cardiol. 1, 785-792 (2005).

13. Steinbrecher, U. P. \& Lougheed, M. Scavenger receptor-independent stimulation of cholesterol esterification in macrophages by low density lipoprotein extracted from human aortic intima. Arterioscler. Thromb. J. Vasc. Biol. 12, 608-625 (1992).

14. Steinberg, D. \& Witztum, J. L. Is the oxidative modification hypothesis relevant to human atherosclerosis? Do the antioxidant trials conducted to date refute the hypothesis? Circulation 105, 2107-2111 (2002).

15. Kim Kyeongdae et al. Transcriptome Analysis Reveals Nonfoamy Rather Than Foamy Plaque Macrophages Are Proinflammatory in Atherosclerotic Murine Models. Circ. Res. 123, 1127-1142 (2018).

16. Fernandez, D. M. et al. Single-cell immune landscape of human atherosclerotic plaques. Nat. Med. 25, 1576-1588 (2019).

17. Zernecke Alma et al. Meta-Analysis of Leukocyte Diversity in Atherosclerotic Mouse Aortas. Circ. Res. 127, 402-426 (2020).

18. Spann, N. J. et al. Regulated accumulation of desmosterol integrates macrophage lipid metabolism and inflammatory responses. Cell 151, 138-152 (2012).

19. Lamarche, B., Lemieux, I. \& Després, J. P. The small, dense LDL phenotype and the risk of coronary heart disease: epidemiology, patho-physiology and therapeutic aspects. Diabetes Metab. 25, 199211 (1999).

20. Smith, E. B. \& Ashall, C. Low-density lipoprotein concentration in interstitial fluid from human atherosclerotic lesions. Relation to theories of endothelial damage and lipoprotein binding. Biochim. Biophys. Acta 754, 249-257 (1983).

21. Kruth, H. S., Huang, W., Ishii, I. \& Zhang, W.-Y. Macrophage foam cell formation with native low density lipoprotein. J. Biol. Chem. 277, 34573-34580 (2002).

22. Goldstein, J. L., Ho, Y. K., Basu, S. K. \& Brown, M. S. Binding site on macrophages that mediates uptake and degradation of acetylated low density lipoprotein, producing massive cholesterol deposition. Proc. Natl. Acad. Sci. U. S. A. 76, 333-337 (1979).

23. Anzinger, J. J. et al. Native low-density lipoprotein uptake by macrophage colony-stimulating factordifferentiated human macrophages is mediated by macropinocytosis and micropinocytosis. Arterioscler. Thromb. Vasc. Biol. 30, 2022-2031 (2010).

24. Goulopoulou, S., McCarthy, C. G. \& Webb, R. C. Toll-like Receptors in the Vascular System: Sensing the Dangers Within. Pharmacol. Rev. 68, 142-167 (2016).

25. Vaeth, M. et al. $\mathrm{Ca}^{2+}$ Signaling but Not Store-Operated $\mathrm{Ca}^{2+}$ Entry Is Required for the Function of Macrophages and Dendritic Cells. J. Immunol. 195, 1202-1217 (2015). 
26. Heil, F. et al. Species-specific recognition of single-stranded RNA via toll-like receptor 7 and 8 . Science 303, 1526-1529 (2004).

27. Tanji, H. et al. Toll-like receptor 8 senses degradation products of single-stranded RNA. Nat. Struct. Mol. Biol. 22, 109-115 (2015).

28. Zhang, S. et al. Small-molecule inhibition of TLR8 through stabilization of its resting state. Nat. Chem. Biol. 14, 58-64 (2018).

29. Jurk, M. et al. Human TLR7 or TLR8 independently confer responsiveness to the antiviral compound R-848. Nat. Immunol. 3, 499 (2002).

30. Diebold, S. S., Kaisho, T., Hemmi, H., Akira, S. \& Reis e Sousa, C. Innate antiviral responses by means of TLR7-mediated recognition of single-stranded RNA. Science 303, 1529-1531 (2004).

31. Liu, J. et al. A five-amino-acid motif in the undefined region of the TLR8 ectodomain is required for species-specific ligand recognition. Mol. Immunol. 47, 1083-1090 (2010).

32. Ishii, N., Funami, K., Tatematsu, M., Seya, T. \& Matsumoto, M. Endosomal localization of TLR8 confers distinctive proteolytic processing on human myeloid cells. J. Immunol. Baltim. Md 1950 193, 5118-5128 (2014).

33. Gorden, K. K. B., Qiu, X. X., Binsfeld, C. C. A., Vasilakos, J. P. \& Alkan, S. S. Cutting edge: activation of murine TLR8 by a combination of imidazoquinoline immune response modifiers and polyT oligodeoxynucleotides. J. Immunol. Baltim. Md 1950 177, 6584-6587 (2006).

34. Allen, R. M. et al. Bioinformatic analysis of endogenous and exogenous small RNAs on lipoproteins. J. Extracell. Vesicles 7, 1506198 (2018).

35. Krieger, M., Brown, M. S., Faust, J. R. \& Goldstein, J. L. Replacement of endogenous cholesteryl esters of low density lipoprotein with exogenous cholesteryl linoleate. Reconstitution of a biologically active lipoprotein particle. J. Biol. Chem. 253, 4093-4101 (1978).

36. Sarvestani, S. T. et al. Sequence-dependent off-target inhibition of TLR7/8 sensing by synthetic microRNA inhibitors. Nucleic Acids Res. 43, 1177-1188 (2015).

37. Robbins, M. et al. 2'-O-methyl-modified RNAs act as TLR7 antagonists. Mol. Ther. J. Am. Soc. Gene Ther. 15, 1663-1669 (2007).

38. Sioud, M., Furset, G. \& Cekaite, L. Suppression of immunostimulatory siRNA-driven innate immune activation by 2'-modified RNAs. Biochem. Biophys. Res. Commun. 361, 122-126 (2007).

39. Mitchell, S. et al. An NFkB Activity Calculator to Delineate Signaling Crosstalk: Type I and II Interferons Enhance NFKB via Distinct Mechanisms. Front. Immunol. 10, (2019).

40. Rayner, K. J. et al. Antagonism of miR-33 in mice promotes reverse cholesterol transport and regression of atherosclerosis. J. Clin. Invest. 121, 2921-2931 (2011).

41. Rahman, S. M. et al. CCAAT/Enhancer-binding Protein $\beta$ (C/EBP $\beta)$ Expression Regulates Dietaryinduced Inflammation in Macrophages and Adipose Tissue in Mice. J. Biol. Chem. 287, 3434934360 (2012). 
42. Zernecke, A. \& Weber, C. Chemokines in atherosclerosis: proceedings resumed. Arterioscler. Thromb. Vasc. Biol. 34, 742-750 (2014).

43. Duewell, P. et al. NLRP3 inflammasomes are required for atherogenesis and activated by cholesterol crystals. Nature 464, 1357-1361 (2010).

44. Patinote, C. et al. Agonist and antagonist ligands of toll-like receptors 7 and 8: Ingenious tools for therapeutic purposes. Eur. J. Med. Chem. 193, 112238 (2020).

45. Janssen, H. L. A. et al. Treatment of HCV infection by targeting microRNA. N. Engl. J. Med. 368, 1685-1694 (2013).

46. Paigen, B., Morrow, A., Holmes, P. A., Mitchell, D. \& Williams, R. A. Quantitative assessment of atherosclerotic lesions in mice. Atherosclerosis 68, 231-240 (1987).

47. Linton, M. F., Atkinson, J. B. \& Fazio, S. Prevention of atherosclerosis in apolipoprotein E-deficient mice by bone marrow transplantation. Science 267, 1034-1037 (1995).

48. Makowski, L. et al. Lack of macrophage fatty-acid-binding protein aP2 protects mice deficient in apolipoprotein E against atherosclerosis. Nat. Med. 7, 699-705 (2001).

49. Babaev, V. R. et al. Macrophage EP4 deficiency increases apoptosis and suppresses early atherosclerosis. Cell Metab. 8, 492-501 (2008).

50. Chen, Y., Yu, Q. \& Xu, C.-B. A convenient method for quantifying collagen fibers in atherosclerotic lesions by ImageJ software. 7.

51. Park, E. K. et al. Optimized THP-1 differentiation is required for the detection of responses to weak stimuli. Inflamm. Res. 56, 45-50 (2007).

52. Fleetwood, A. J., Lawrence, T., Hamilton, J. A. \& Cook, A. D. Granulocyte-Macrophage ColonyStimulating Factor (CSF) and Macrophage CSF-Dependent Macrophage Phenotypes Display Differences in Cytokine Profiles and Transcription Factor Activities: Implications for CSF Blockade in Inflammation. J. Immunol. 178, 5245-5252 (2007).

53. Na, Y. R., Jung, D., Gu, G. J. \& Seok, S. H. GM-CSF Grown Bone Marrow Derived Cells Are Composed of Phenotypically Different Dendritic Cells and Macrophages. Mol. Cells 39, 734 (2016).

54. Martin, M. Cutadapt removes adapter sequences from high-throughput sequencing reads. EMBnet.journal 17, 10-12 (2011).

55. Dobin, A. et al. STAR: ultrafast universal RNA-seq aligner. Bioinforma. Oxf. Engl. 29, 15-21 (2013).

56. Liao, Y., Smyth, G. K. \& Shi, W. featureCounts: an efficient general purpose program for assigning sequence reads to genomic features. Bioinforma. Oxf. Engl. 30, 923-930 (2014).

57. Love, M. I., Huber, W. \& Anders, S. Moderated estimation of fold change and dispersion for RNA-seq data with DESeq2. Genome Biol. 15, 550 (2014).

58. Langmead, B., Trapnell, C., Pop, M. \& Salzberg, S. L. Ultrafast and memory-efficient alignment of short DNA sequences to the human genome. Genome Biol. 10, R25 (2009).

59. Galkina, E. et al. Lymphocyte recruitment into the aortic wall before and during development of atherosclerosis is partially L-selectin dependent. J. Exp. Med. 203, 1273-1282 (2006). 
60. Liu, Q. et al. scRNABatchQC: multi-samples quality control for single cell RNA-seq data. Bioinforma. Oxf. Engl. 35, 5306-5308 (2019).

61. Stuart, T. et al. Comprehensive Integration of Single-Cell Data. Cell 177, 1888-1902.e21 (2019).

62. Franzén, O., Gan, L.-M. \& Björkegren, J. L. M. PanglaoDB: a web server for exploration of mouse and human single-cell RNA sequencing data. Database J. Biol. Databases Curation 2019, (2019).

63. Audzevich, T. et al. Pre/pro-B cells generate macrophage populations during homeostasis and inflammation. Proc. Natl. Acad. Sci. U. S. A. 114, E3954-E3963 (2017).

64. Williams, J. W. et al. Limited proliferation capacity of aortic intima resident macrophages requires monocyte recruitment for atherosclerotic plaque progression. Nat. Immunol. 21, 1194-1204 (2020).

65. Tacke, R. et al. The transcription factor NR4A1 is essential for the development of a novel macrophage subset in the thymus. Sci. Rep. 5, 10055 (2015).

66. Sarrazy, V. et al. Maintenance of Macrophage Redox Status by ChREBP Limits Inflammation and Apoptosis and Protects against Advanced Atherosclerotic Lesion Formation. Cell Rep. 13, 132-144 (2015).

67. Robinson, M. D., McCarthy, D. J. \& Smyth, G. K. edgeR: a Bioconductor package for differential expression analysis of digital gene expression data. Bioinforma. Oxf. Engl. 26, 139-140 (2010).

68. Michell, D. L. et al. Isolation of High-density Lipoproteins for Non-coding Small RNA Quantification. JoVE J. Vis. Exp. e54488 (2016) doi:10.3791/54488.

69. Greenwald, R. A. CRC Handbook of Methods for Oxygen Radical Research. (CRC Press, 1987).

\section{Figures}



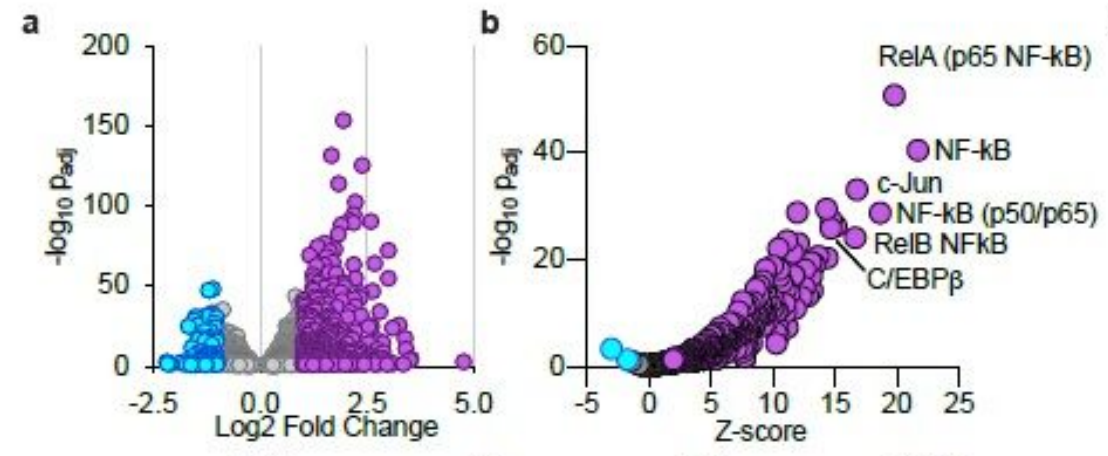

d
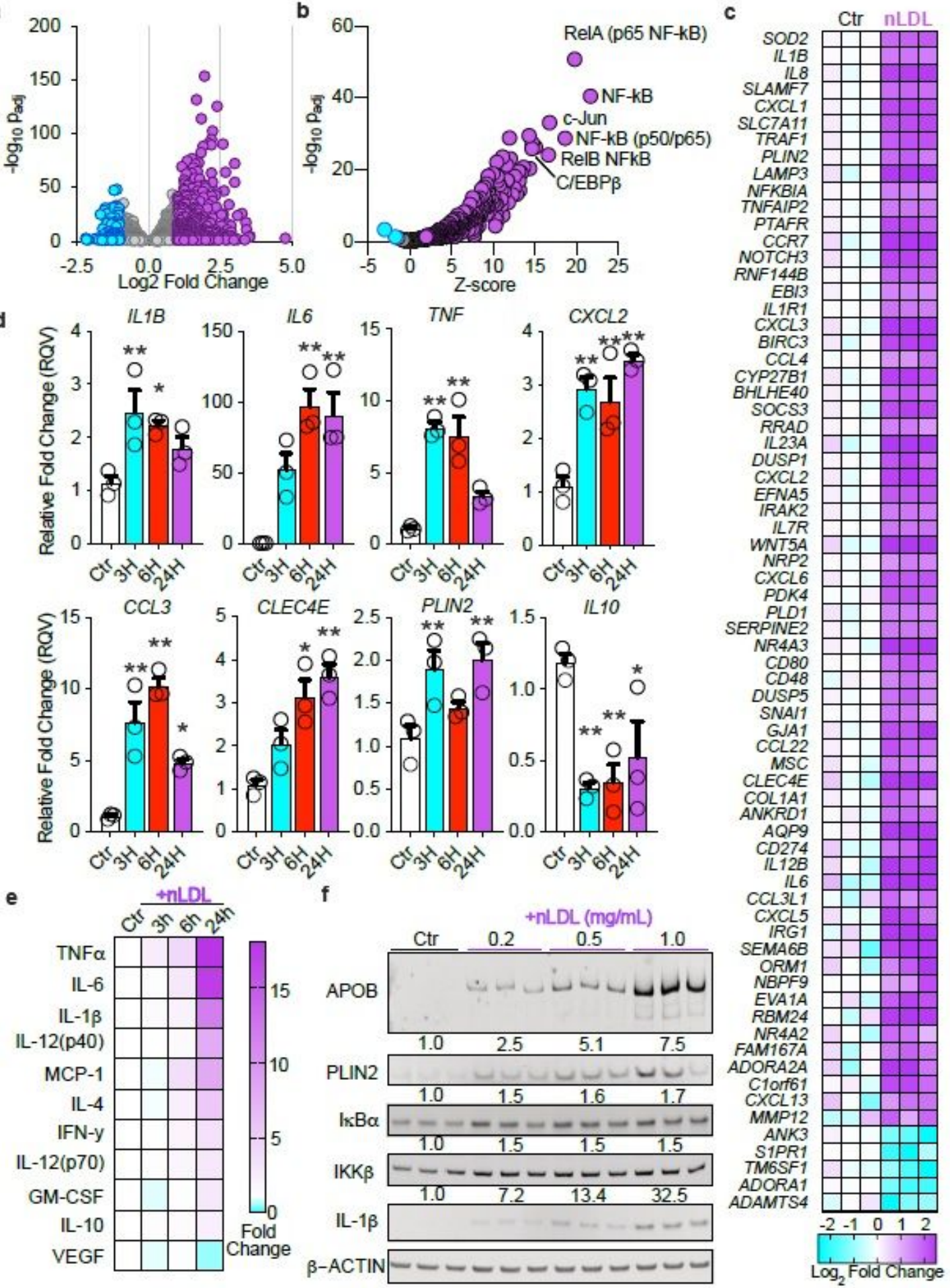

\section{Figure 1}

Native LDL ( $\mathrm{nLDL}$ ) induce inflammatory activation in macrophages. (a) Differentially altered transcripts (mRNA) of THP-1 macrophages treated with $0.5 \mathrm{mg} / \mathrm{ml} \mathrm{nLDL}$ for $24 \mathrm{~h}$ identified by RNA-seq $(\mathrm{n}=3)$. (b) Transcription factor network enrichment analysis of $n L D L$-altered genes. (c) Differentially expressed transcripts of the RelA (NF-kB p65) cluster. (d) mRNA expression and (e) secreted cytokine levels of selected targets of NF-KB and C/EBPb following exposure to $0.5 \mathrm{mg} / \mathrm{ml} \mathrm{nLDL}$ for $3 \mathrm{~h}, 6 \mathrm{~h}$, $24 \mathrm{~h}$ relative to 
cells receiving no treatment for $24 \mathrm{~h}(\mathrm{Ctr})(\mathrm{n}=3)$. (f) Immunoblots of selected proteins of THP-1 cells exposed to $\mathrm{nLDL}$ for $24 \mathrm{~h}$. Data are mean +/- SEM. One-way ANOVA, Benjamini, Krieger and Yekutieli FDR $(\mathrm{Q}=0.05) .{ }^{*} \mathrm{p}<0.05,{ }^{* *} \mathrm{p}<0.01$.
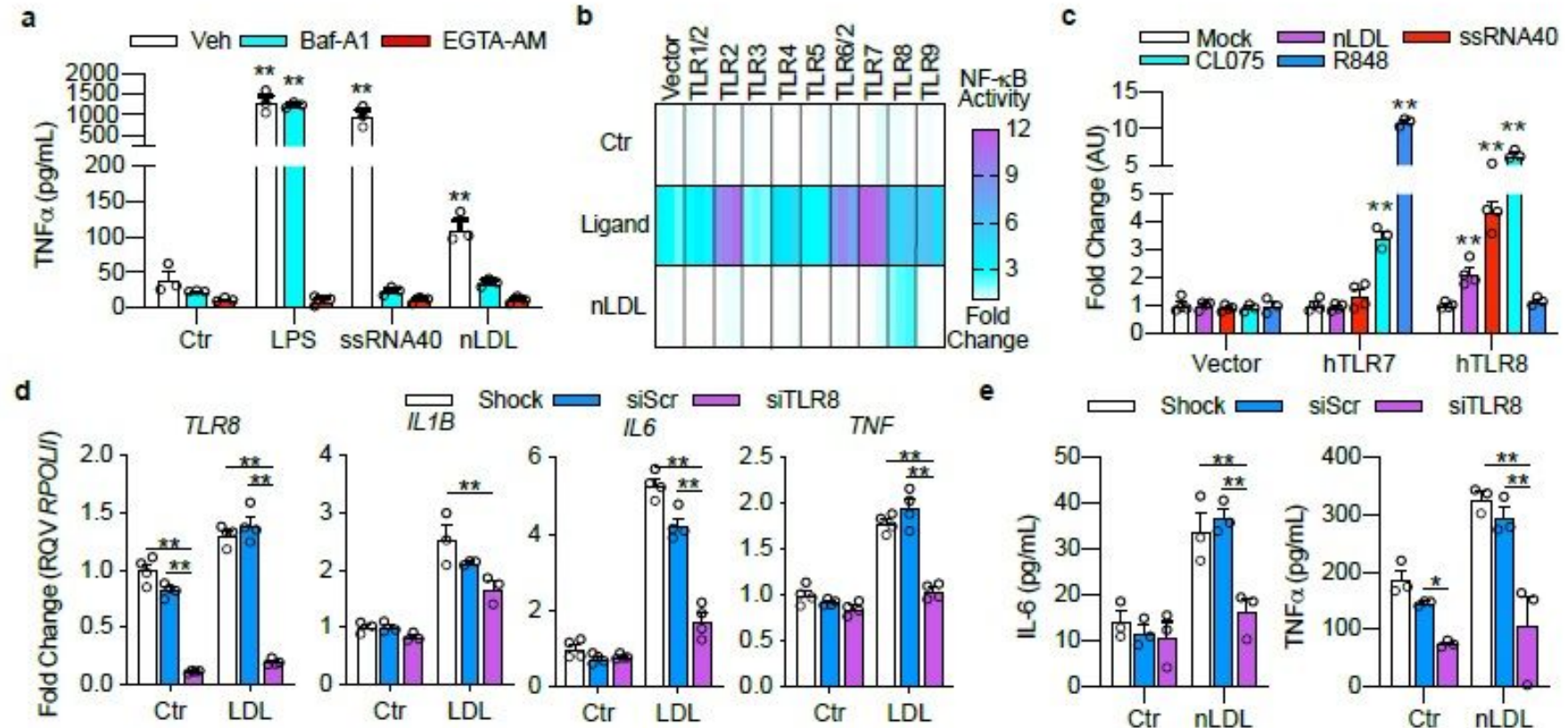

$\mathbf{f}$
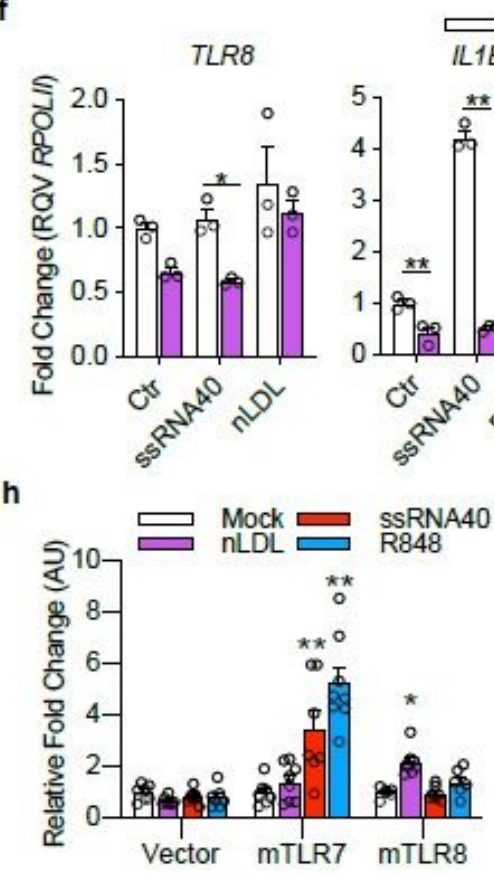

$\coprod_{\text {IL1B }} \mathrm{Ctr} \rightleftharpoons \underset{\text { IL6 }}{\text { CU-CPT9a }}$
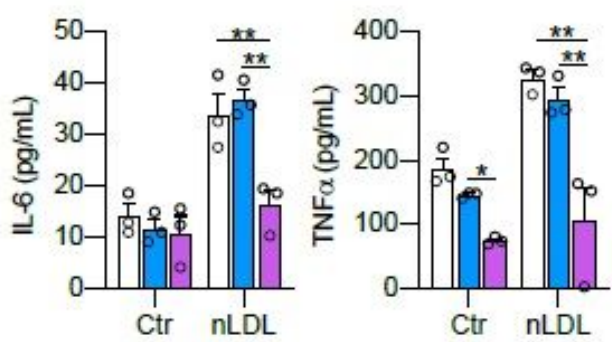

g

C Ctr $\square$ CU-CPT9a
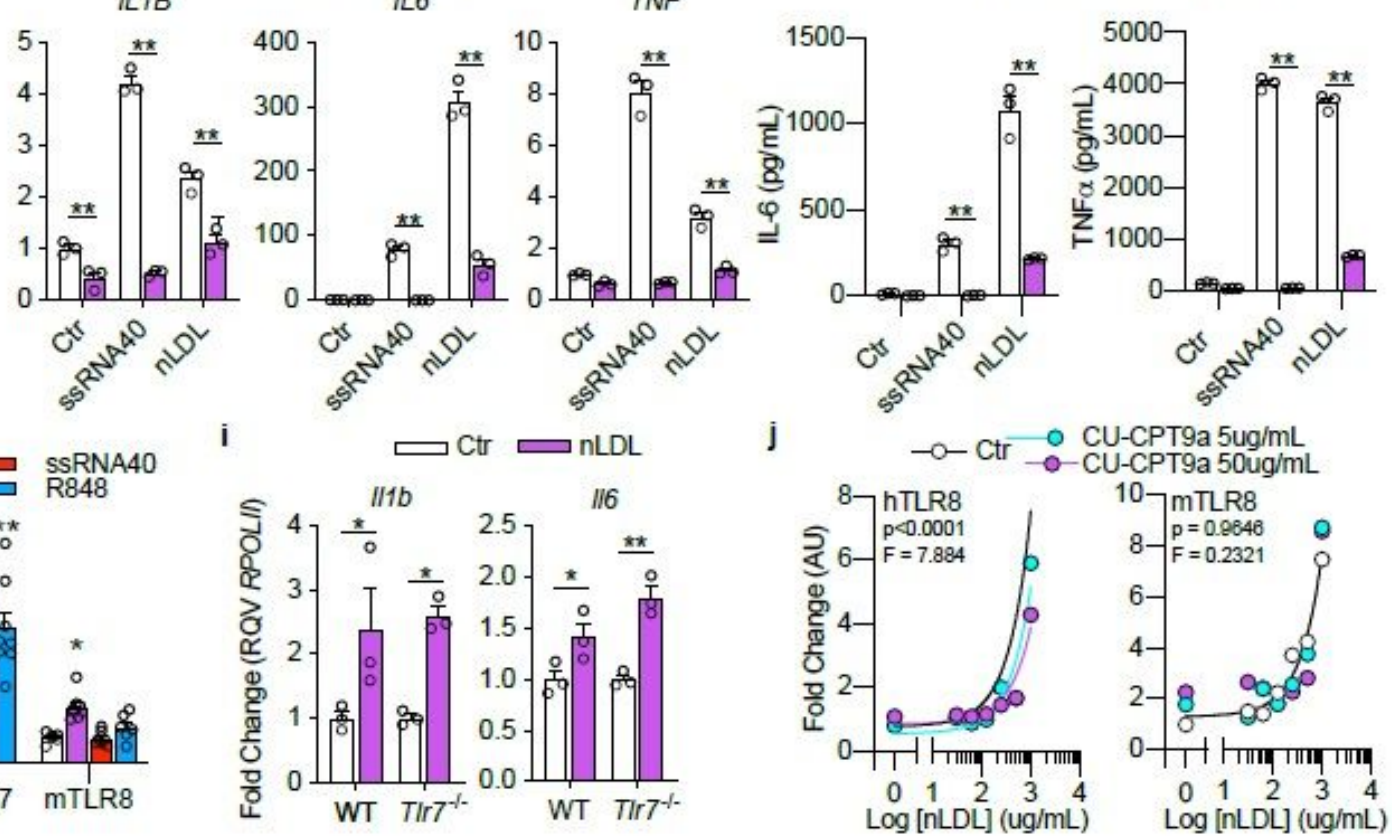

\section{Figure 2}

Native LDL activates NF-KB through TLR8 in macrophages. (a) TNF-a secretion of THP-1 macrophages pre-treated with vehicle (Veh; DMSO), bafilomycin A1 (Baf-A1; $500 \mu \mathrm{M}$ ) or EGTA-AM for $45 \mathrm{~min}$, followed by exposure to LPS $(100 \mathrm{ng} / \mathrm{mL})$, ssRNA40 $(1 \mathrm{mg} / \mathrm{mL}), \mathrm{nLDL}(0.5 \mathrm{mg} / \mathrm{ml})$ or untreated $(C t r)$ for $6 \mathrm{~h}(\mathrm{n}=3)$. (b-c) Normalized NF-KB-driven luciferase activity of HEK293T cells over-expressing an empty vector, 
individual or tandem human TLRs following treatments with vehicle (Ctr), cognate TLR ligand(s), or nLDL $(0.5 \mathrm{mg} / \mathrm{ml})(\mathrm{n} \geq 3)$. (d) mRNA expression and (e) cytokine secretion of THP-1 macrophages electroporated with scrambled (siScr) or TLR8-targeted (siTLR8) siRNAs or without siRNA (shock) following $24 \mathrm{~h}$ of $\mathrm{nLDL}$ exposure $(0.5 \mathrm{mg} / \mathrm{ml})(\mathrm{n}=4)$. (f) mRNA expression and $(\mathrm{g})$ cytokine secretion of THP-1 macrophages pre-treated with CU-CPT9a followed by 24h of $n L D L$ exposure $(n=3)$. $(h)$ Normalized NF-KB-driven luciferase activity of HEK293T cells over-expressing an empty vector, murine TLR7 or murine TLR8 following treatment with a mock transfection, R848 (10 mM), ssRNA40 $(2 \mathrm{mg} / \mathrm{mL})$, or $\mathrm{nLDL}(0.5 \mathrm{mg} / \mathrm{ml})$ for $24 \mathrm{~h}(\mathrm{n} \geq 6)$. (i) Relative expression of wild-type (WT) and TIr7-deficient BMDMs following treatment with $0.5 \mathrm{mg} / \mathrm{ml} \mathrm{nLDL}$, or $1 \mathrm{mg} / \mathrm{mL}$ ssRNA40 for $24 \mathrm{~h}(\mathrm{n}=3)$. (j) Dose-response curves for nLDL-induced luciferase activity in HEK293T cells over-expressing human or murine TLR8, with or without CU-CPT9a pre-treatment $(\mathrm{n}=3) .2 \mathrm{a}-2 \mathrm{~h}$, Two-way ANOVA, Benjamini, Krieger and Yekutieli FDR $(\mathrm{Q}=0.05)$, Data are mean +/-SEM. 2l, Mann-Whitney test (two-sided). 2j: Non-linear regression with extra sum-of-squares $F$ test. ${ }^{*} p<0.05,{ }^{* *} p<0.01$. 
a

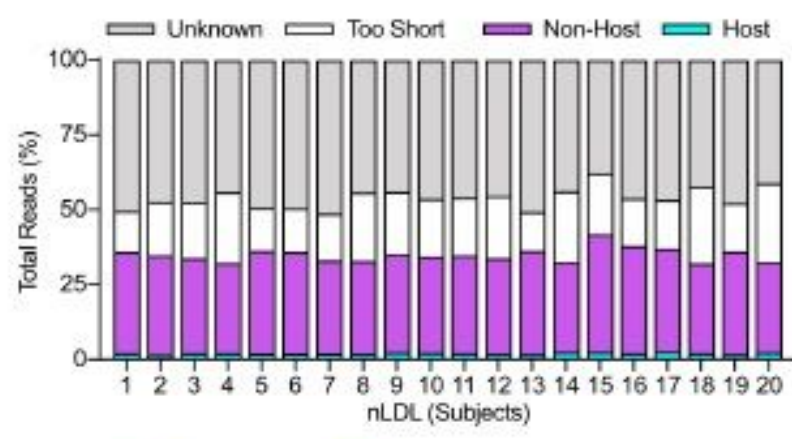

c

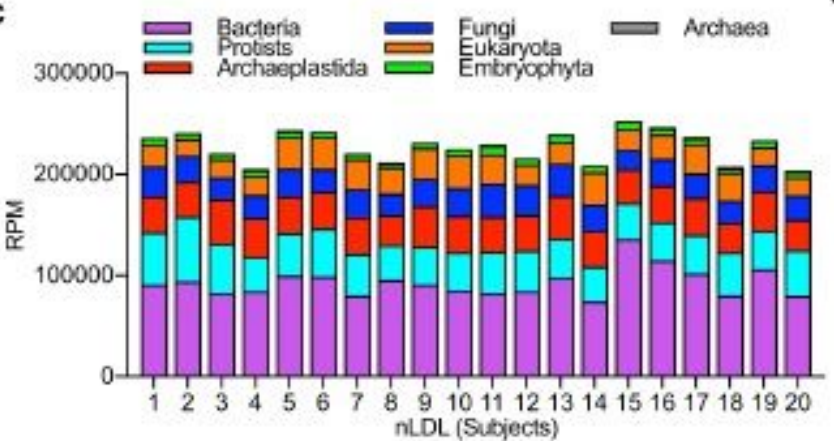

b

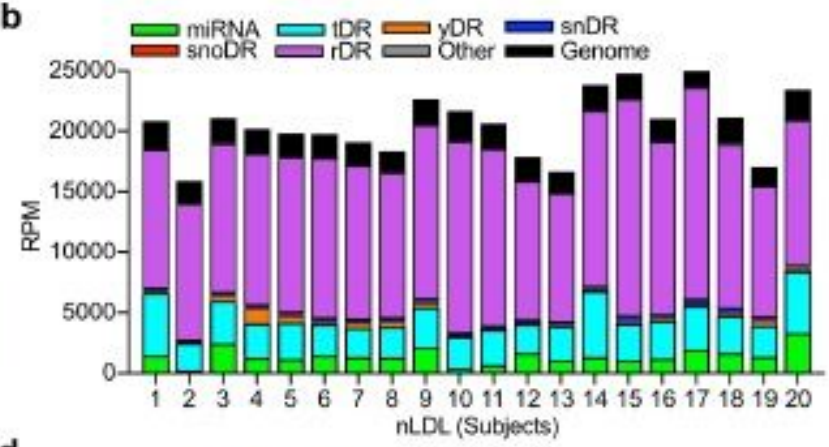

d

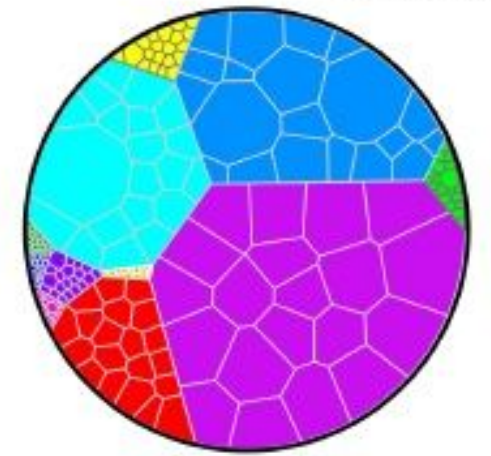

Phyla

Actinobacteria

- Bacteroidetes

1 Cyanobacteria

Euryarchaeota

Firmicutes

ㅁusobacteria

- Proteobacteria

- Spirochaetes

- Synergistetes

- Tenericutes

- Vemucomicrobia

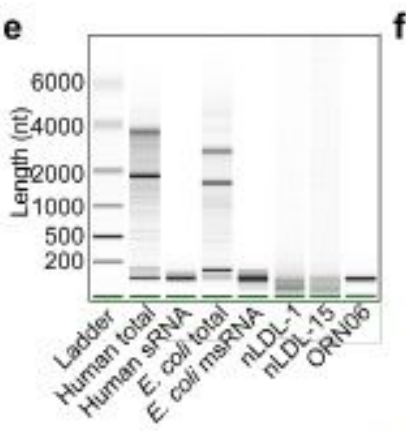

t i

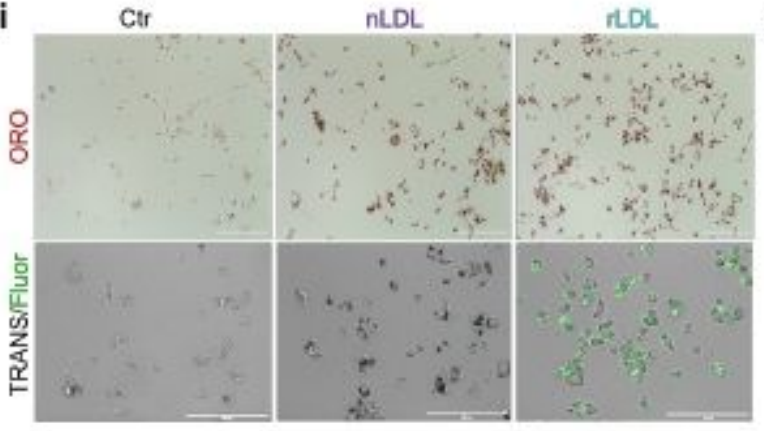

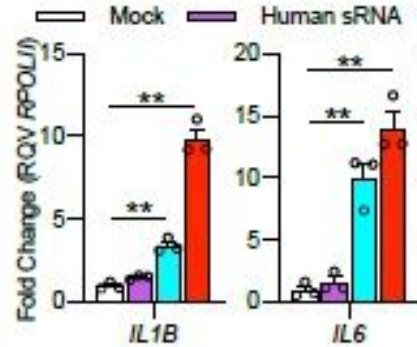

IL6

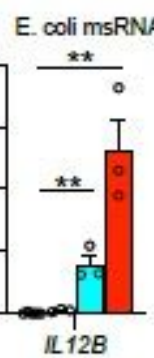

j
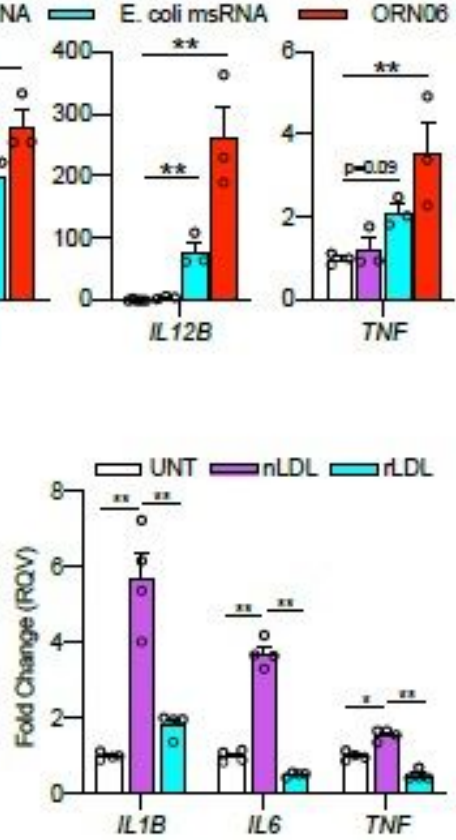

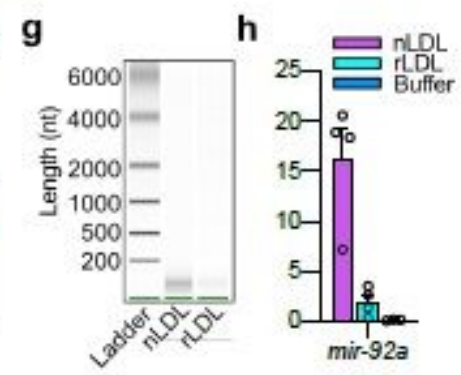

k

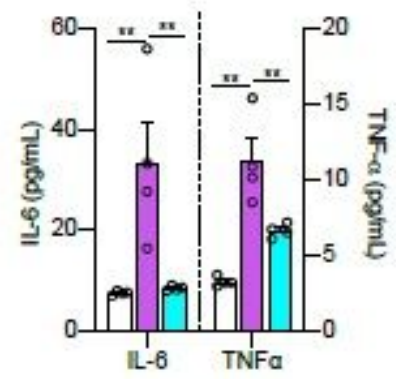

\section{Figure 3}

LDL are enriched with microbial sRNA that confer inflammatory potential. (a) Relative proportion of reads identified as endogenous, exogenous, unknown, or too short for conservative mapping with TIGER. (b) Endogenous sRNA abundance quantified as reads per million total reads (RPM). (c) Alignment of nonhuman sRNAs to the SILVA ribosomal RNA database and grouped by taxa. (d) Voronoi plot representing relative composition of bacterial phyla identified by mapping to genomes of the human microbiome database. (e) Total RNA and sRNA isolated from human macrophages (THP-1) and bacteria (E. coli), as well as, total RNA isolated from selected nLDL subjects of (a-d) and ORN06 (20-mer) were assessed by 
Bioanalyzer (PicoChip). (f) mRNA expression of THP-1 macrophages transfected with $1 \mathrm{mg} / \mathrm{ml}$ of isolated human sRNA, bacterial sRNA or ORN06 $(\mathrm{n}=3)$. (g) Total RNA of $\mathrm{nLDL}$ and rLDL assessed by Bioanalyzer. (h) Quantification of representative miRNA across independent preparations of rLDL and matched $n L D L$, or buffer negative control $(n=4)$. (i) Oil-Red-O staining and fluorescence microscopy. Scale bar $=200 \mathrm{~mm}(\mathrm{j})$ mRNA expression, and (k) cytokine secretion of THP-1 cells after treatment with $0.5 \mathrm{mg} / \mathrm{ml} \mathrm{nLDL}$ or rLDL for 24h $(\mathrm{n}=4)$. Two-way ANOVA, Benjamini, Krieger and Yekutieli FDR $(\mathrm{Q}=0.05)$. Data are mean $+/$-SEM. ${ }^{\star \star} \mathrm{p}<0.01$.
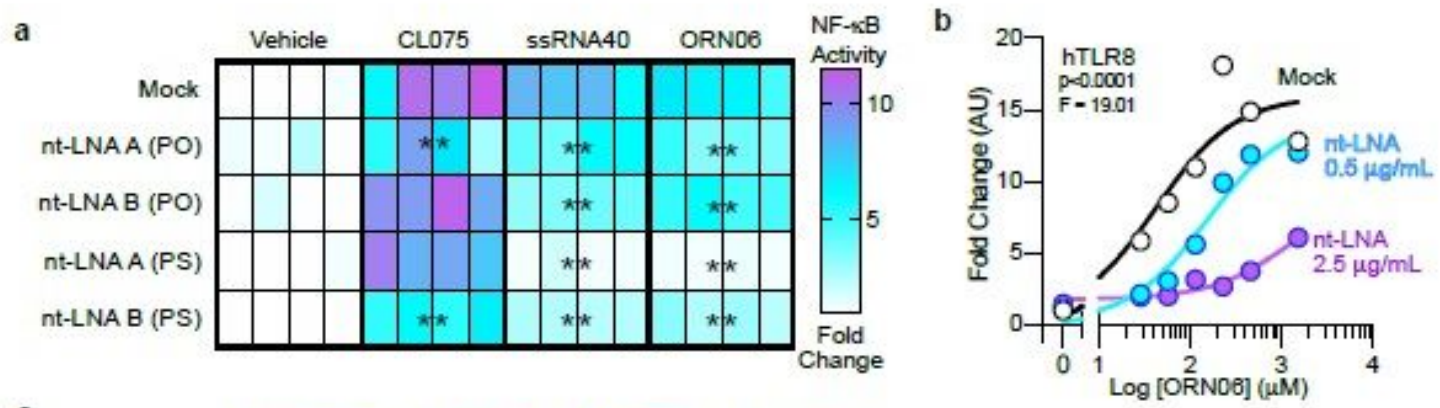

C
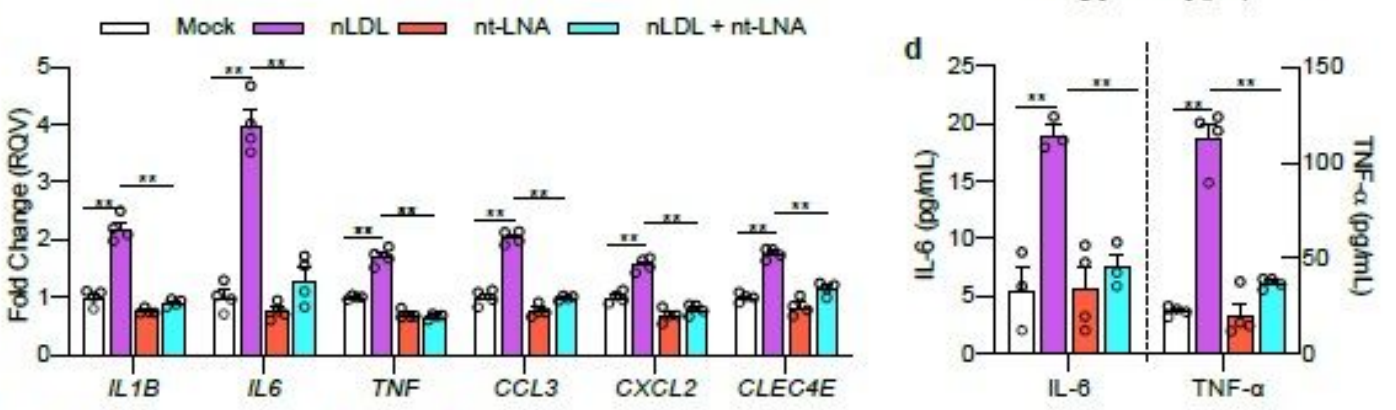

e

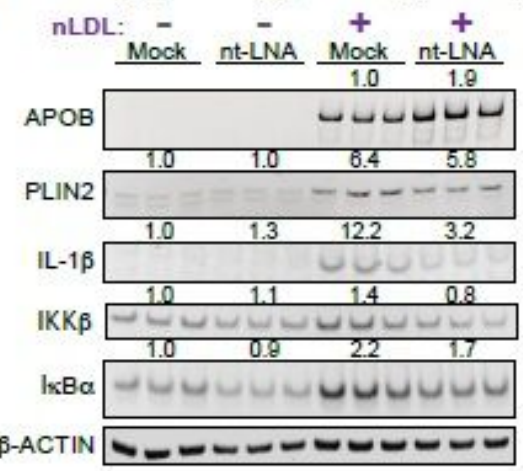

h
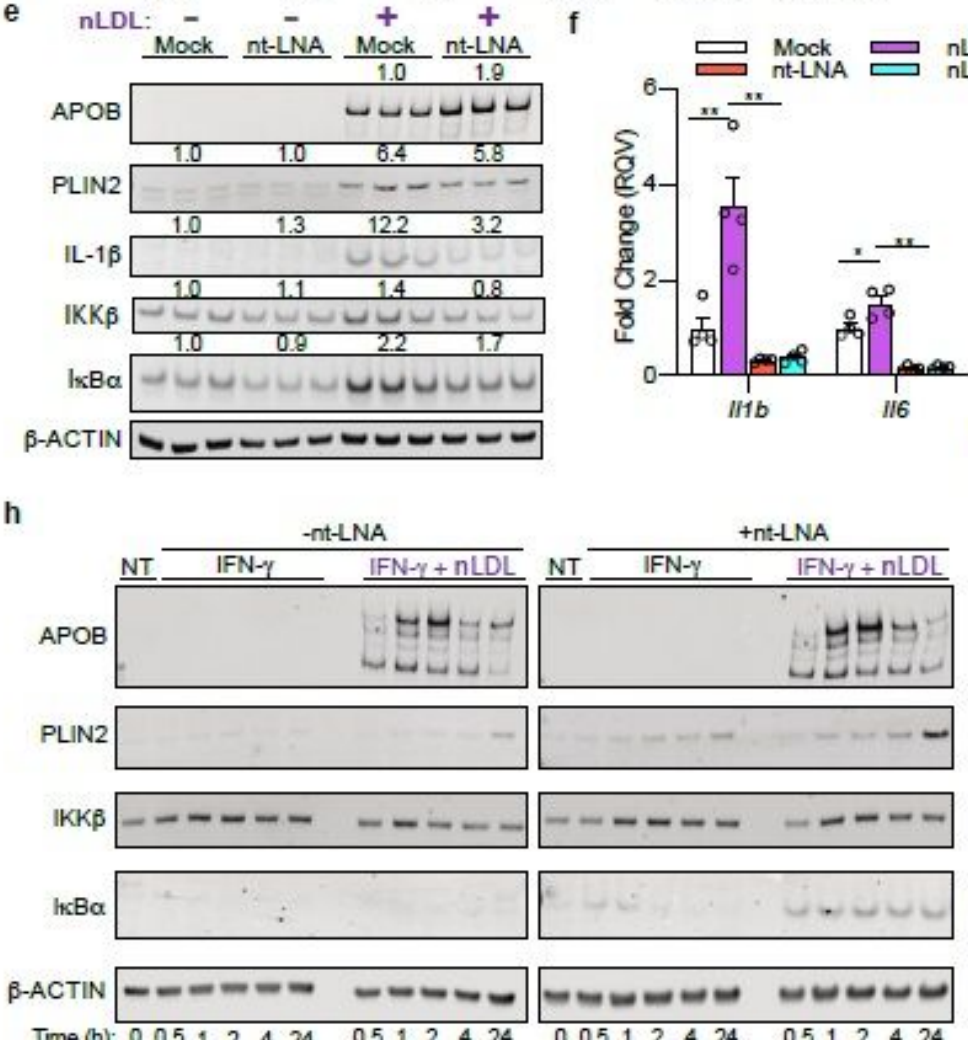

Time (h): $\begin{array}{llllllllllll}0 & 0.5 & 1 & 2 & 4 & 24 & 0.5 & 1 & 2 & 4 & 24\end{array}$

\begin{tabular}{lllllllllll}
\hline & 0.51 & 2 & 4 & 24 & 0.51 & 2 & 4 & 24
\end{tabular}

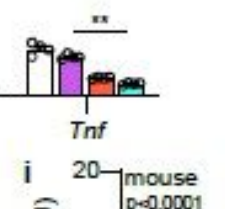

g
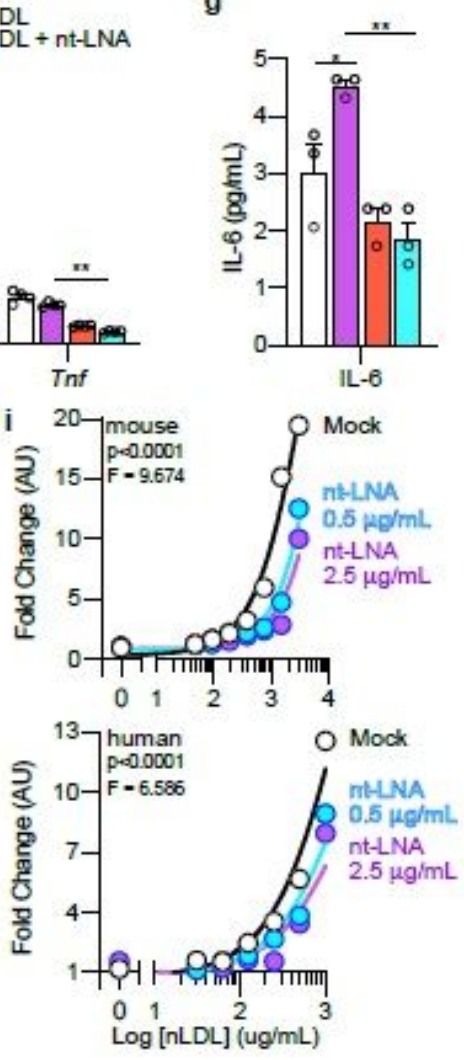


\section{Figure 4}

Non-targeting locked-nucleic acids are competitive antagonists of TLR8. (a) NF-KB driven luciferase activity of HEK293T cells over-expressing human TLR8 and treated with agonists CL075 $(2.5 \mathrm{mg} / \mathrm{ml})$, ssRNA40 $(2 \mathrm{mg} / \mathrm{ml})$, and ORN06 $(2 \mathrm{mg} / \mathrm{mL})$, in the presence or absence of $2 \mathrm{mg} / \mathrm{mL}$ nt-LNA's harboring phosphodiester (PO) or phosphorothioate (PS) backbones $(n=4)$. Statistical comparison of mock vs ntLNA. (b) Dose-response curves for ORN06-induced luciferase activity in HEK293T cells over-expressing human TLR8, with or without nt-LNA $(n=3)$. (c) mRNA expression, (d) cytokine secretion and (e) immunoblotting of THP-1 macrophages following $24 \mathrm{~h}$ treatment with or without $0.5 \mathrm{mg} / \mathrm{ml} \mathrm{nLDL}$ in the presence or absence of $2.5 \mathrm{mg} / \mathrm{mL} \mathrm{nt}-\mathrm{LNA}(\mathrm{n} \geq 3$ ). (f) mRNA expression, (g) cytokine secretion and (h) immunoblotting of BMDMs following up to $24 \mathrm{~h}$ treatment with IFN-g $(100 \mathrm{U} / \mathrm{mL})+/-0.5 \mathrm{mg} / \mathrm{ml} \mathrm{nLDL}$ in the presence or absence of $2.5 \mathrm{mg} / \mathrm{mL}$ nt-LNA $(n \geq 3)$. (i) Dose-response curves for $n L D L$-induced luciferase activity in HEK293T cells over-expressing murine TLR8 and TLR7, or human TLR8, +/- pretreatment with nt-LNA $(\mathrm{n}=3) .4 \mathrm{a}, 4 \mathrm{c}, 4 \mathrm{e}, 4 \mathrm{f}$ Two-way ANOVA, or $4 \mathrm{~g}$ One-way ANOVA, Benjamini, Krieger and Yekutieli FDR (Q=0.05). 4b, 4i: Non-linear regression with extra sum-of-squares $F$ test. Data are mean +/SEM. ${ }^{*} \mathrm{p}<0.05,{ }^{* *} \mathrm{p}<0.01$ 

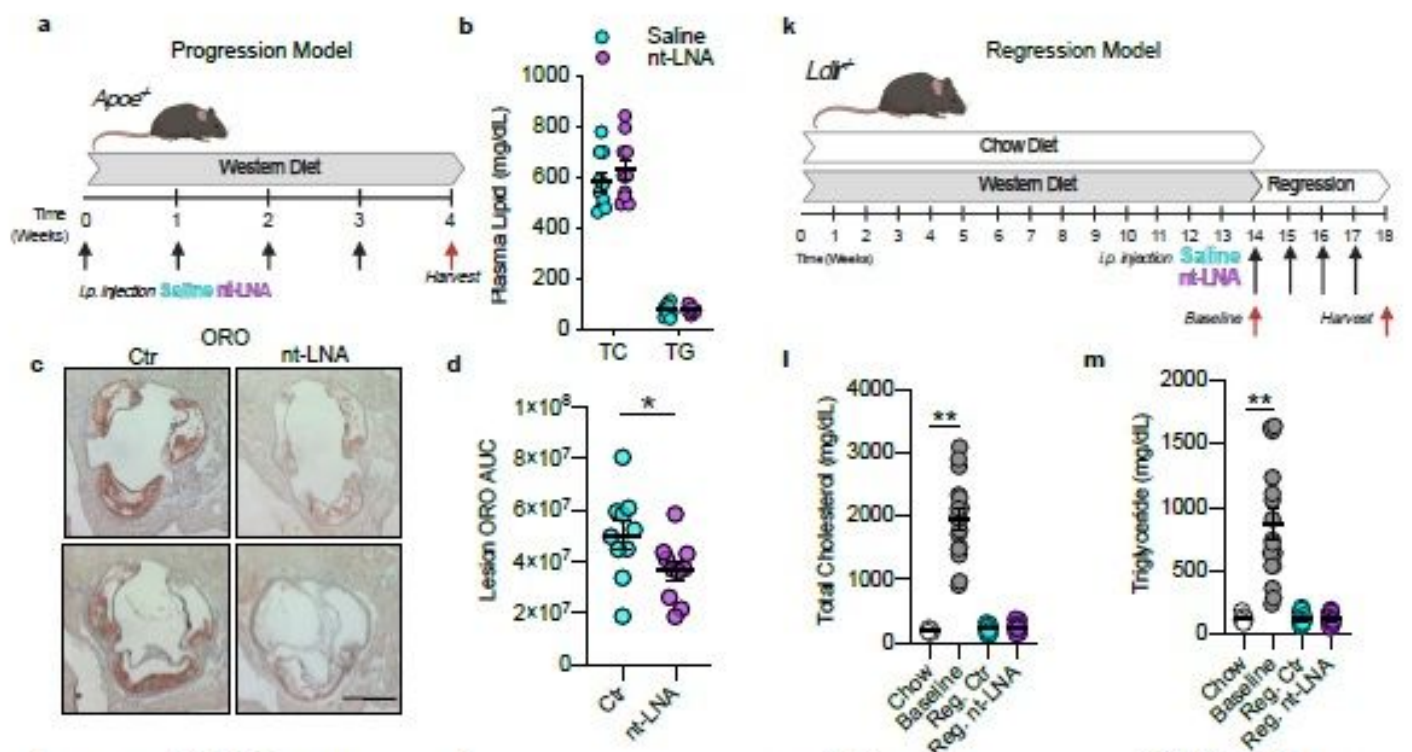

m
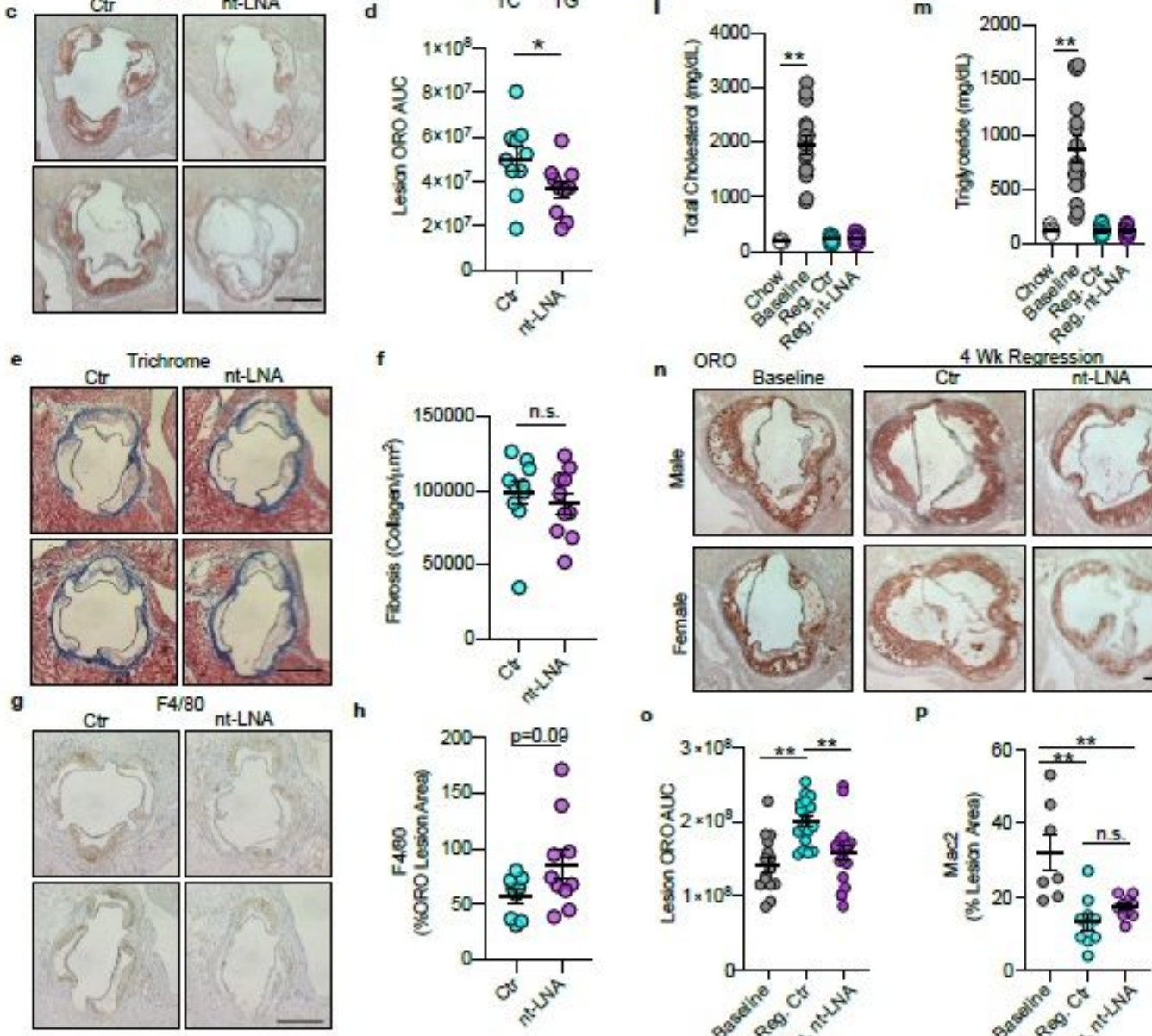

$\circ$
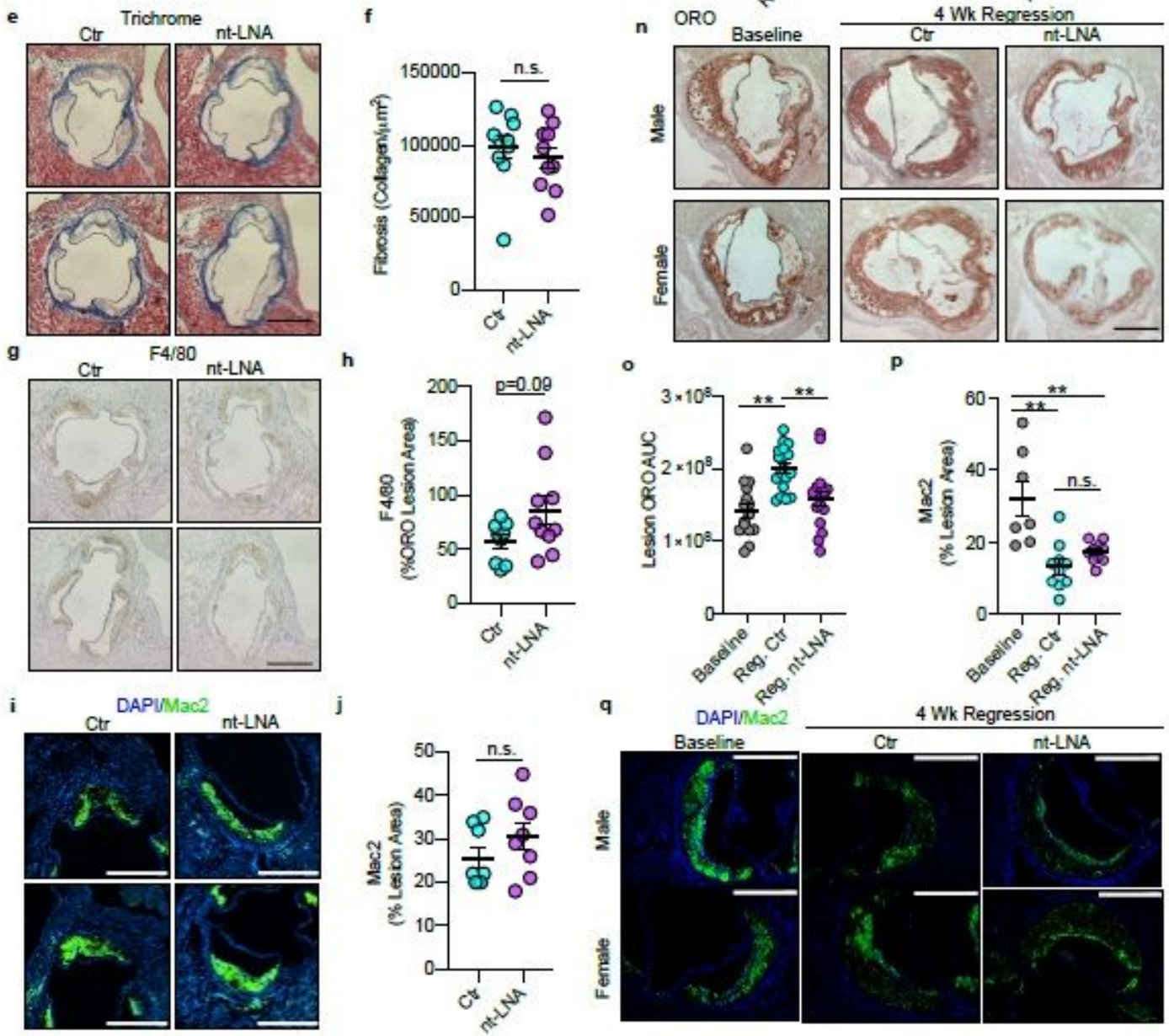

q
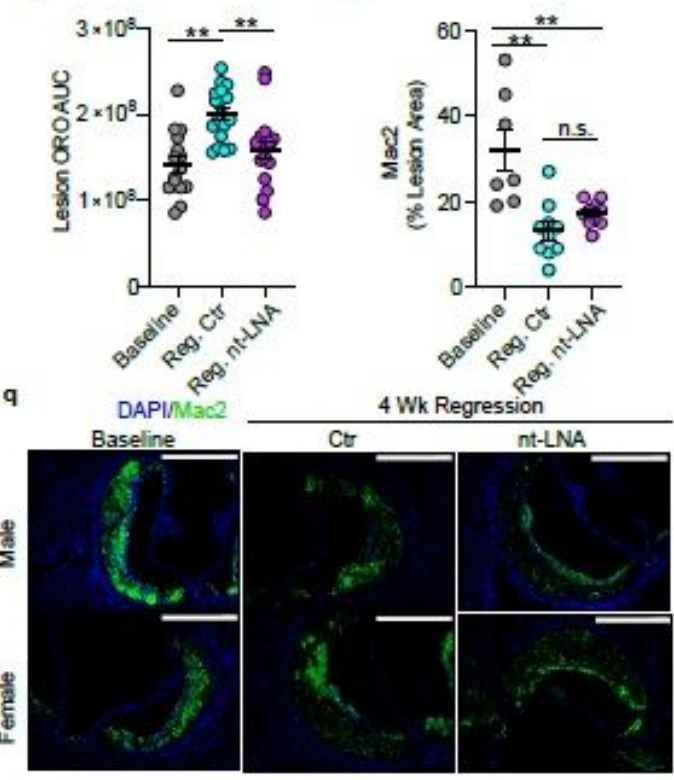

\section{Figure 5}

Non-targeting locked-nucleic acids reduce atherosclerosis in mice. (a) Schematic of progression study design. Apoe-/- mice, fed an atherogenic diet for 4 weeks, were injected once weekly with saline control (Ctr; $n=10)$ or nt-LNA (30 mg/kg; n=10). (b) Plasma lipids at time of sacrifice. (c-d) Lesion area under the curve (AUC), as determined by Oil-Red-O (ORO) staining in the aortic root. (e-f) Quantification of lesion fibrosis by Masson's Trichrome staining. (g-h) Quantification of F4/80+ macrophage content in the lesion 
by immunohistochemistry. (i-j) Quantification of Mac2+ macrophage content in the lesion by immunofluorescence microscopy. (k) Schematic for regression study design. Ldlr-/- mice fed atherogenic diet for 14 weeks, switched to chow diet and injected once weekly with saline control (Ctr) or nt-LNA (30 mg/kg). $(\mathrm{l}, \mathrm{m})$ Plasma lipid levels at baseline (chow, $n=8$; atherogenic diet $n=15)$ and at diet intervention with saline control (Reg. Ctr; $n=18$ ) or nt-LNA (Reg. nt-LNA; $n=17$ ) treatments. ( $n-0)$ Lesion area under the curve (AUC), as determined by ORO staining in the aortic root. ( $p-q)$ Quantification of Mac2+ macrophages by immuno-fluorescence microscopy. Scale bar $=500 \mathrm{~mm}$. 5b,5c,5e,5g,5i, Mann-Whitney test (two-sided); 5l, $5 \mathrm{~m}, 5 \mathrm{n}, 5 \mathrm{p}$ One-way ANOVA Sidak's post-hoc. Data are mean $+/$-SEM. ${ }^{*} \mathrm{p}<0.05,{ }^{*} \mathrm{p}<0.01$ 

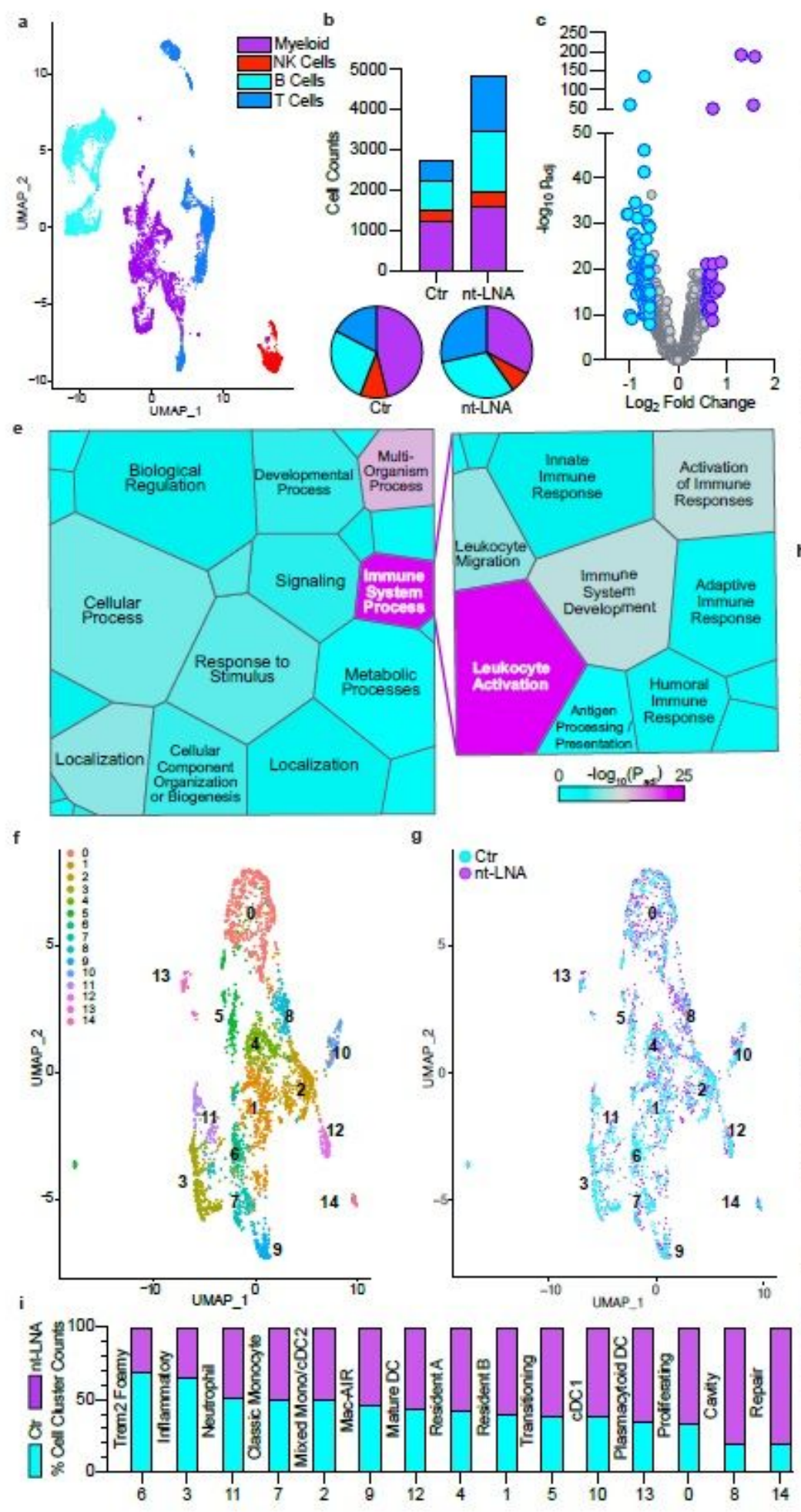
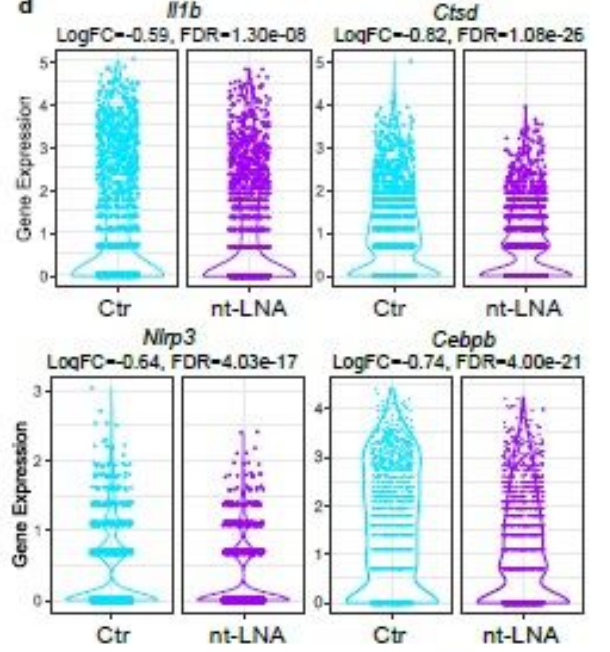

h

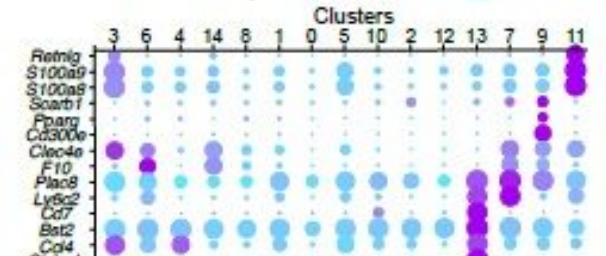

che
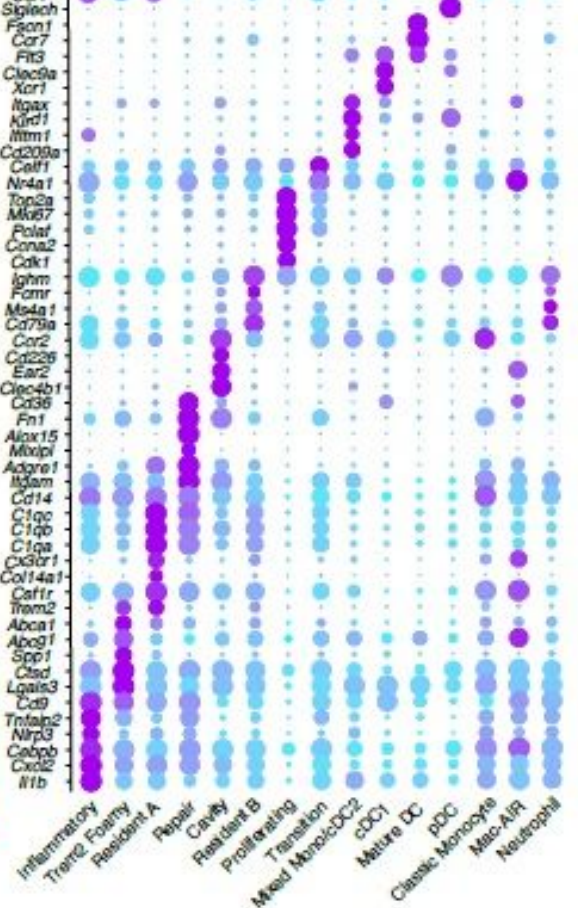

\section{Figure 6}

Non-templated locked-nucleic acids alter macrophages phenotypes within the atherosclerotic lesion. (a) Uniform manifold approximation and projection (UMAP) of single-cell RNA sequencing analysis of fluorescence-activated cell sorted CD45+CD3- immune cells from atherosclerotic lesions of Apoe-/- mice fed a atherogenic diet and treated with saline control (Ctr) or nt-LNA for four weeks. (b) Absolute (histogram) and relative (pie) quantification of immune cell types obtained from (a). (c) Differential 
expression analysis between myeloid cells obtained from each treatment group. (d) Violin plots of selected transcripts found to be differentially altered in (c). (e) Voronoi plots of gene ontology analysis (left) and detailed expansion of key segments of the immune system process (right). (f,g) UMAP projections of macrophage re-clusters (f) and source (g) of macrophages re-clustered from (a). (h) Relative expression (color) and \% of cells reaching the threshold of detection (size) for selected transcripts enriched for each cluster of (f). Relative contribution of each treatment to clusters obtained from (f).

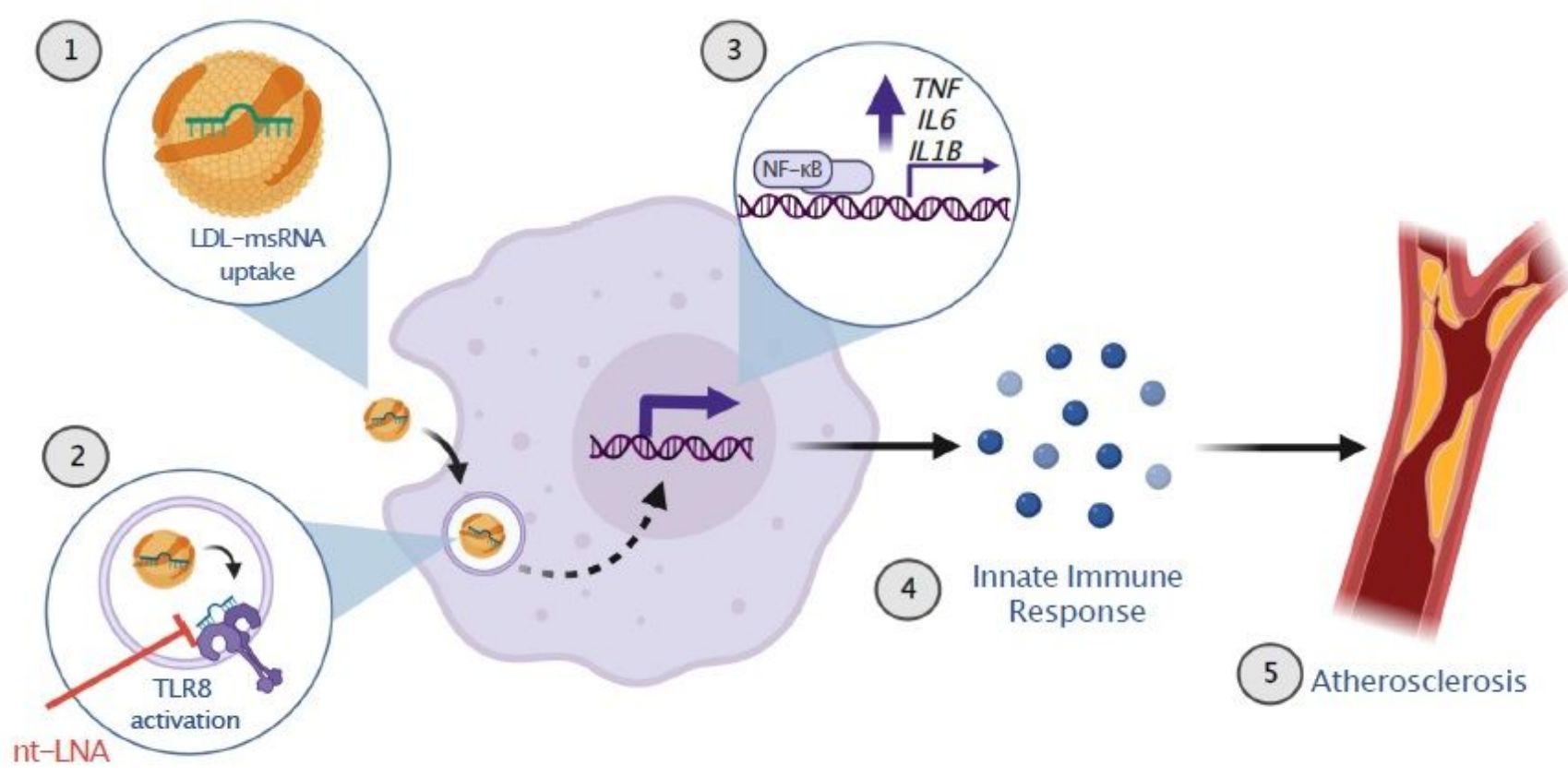

\section{Figure 7}

Proposed mechanism for LDL-msRNA in atherosclerosis-associated inflammation. 1) Native LDL with associated msRNA cargo is internalized and catabolized within the endosomal network. 2) Released msRNA cargo during catabolism is recognized by endosomal TLR8, which can be out-competed by chemically modified oligonucleotides (e.g. nt-LNA). 3) Activation of TLR8 promotes translocation of NF$\mathrm{kB}$ to the nucleus and up-regulation of target genes (e.g. TNF, IL6, IL1B) and 4) release of cytokines that initiate an innate immune response. 5) Sustained LDL-msRNA-driven inflammation promotes atherosclerotic plaque progression and impedes resolution.

\section{Supplementary Files}

This is a list of supplementary files associated with this preprint. Click to download.

- AllenMSTablesv1copy.xlsx

- SupplementNCB.pdf 